\title{
Approximate parametrization of plane algebraic curves by linear systems of curves
}

\author{
Sonia Pérez-Díaz ${ }^{\mathrm{a}}$, J. Rafael Sendra ${ }^{\mathrm{a}}$, Sonia L. Rueda ${ }^{\mathrm{b}}$, Juana Sendra ${ }^{\mathrm{c}}$ \\ a Dpto. de Matemáticas, Universidad de Alcalá, E-28871 Madrid, Spain \\ b Dpto. de Matemática Aplicada, E.TS. Argtitecttira, Universidad Politécifica de Madrid, E-28040 Madrid, Spain \\ 'Dpto. de Matematica Aplfcada a la I.T. de Telecomunicacion, E.U.I. Teleconiunifación, Universidad Polftécrica de Madrid, E-28031 Madrid, Spain
}

Keyword:

Approximate

Parametrization

Plane algebraic curves

Linear systems of curves

\begin{abstract}
A B S T R A C T
It is well known that an irreducible algebraic curve is rational (i.e. parametric) if and only if its genus is zero. In this paper, given a tolerance $\epsilon>0$ and an $\epsilon$-irreducible algebraic affine plane curve $\mathcal{C}$ of proper degree $d$. We introduce the notion of $t$-rationality, and we provide an algorithm to parametrize approximately affine $\epsilon$-rational plane curves by means of linear systems of $(d-2)$-degree curves. The algorithm outputs a rational parametrization of a rational curve $\bar{c}$ of degree $d$ which has the same points at infinity as $c$. Moreover. although we do not provide a theoretical analysis. our empirical analysis shows that $\overline{\mathcal{C}}$ and $c$ are close in practice.
\end{abstract}

\section{Introduction}

Let $\mathfrak{O}^{*}$ be an algebraic or geometric object that satisfies a property $\mathfrak{P}$ that implies the existence of certain associated objects $\mathfrak{O}_{j}^{*}$; for instance, $\mathfrak{O}^{*}$ might be a polynomial, $\mathfrak{P}$ the fact of being reducible and $\mathfrak{O}_{j}^{*}$ the irreducible factors. Computer algebra techniques provide, for a wide class of situations, algorithms to check $\mathfrak{P}$, and to compute exactly the associated objects $\mathfrak{O}_{1}^{*}$. However, in many practical applications, we receive a perturbation $\mathfrak{O}$ of $\mathfrak{O}^{*}$, where $\mathfrak{P}$ does not hold anymore neither the associated objects $\mathfrak{O}_{i}^{*}$ exist. The problem, then, consists in computing a new object $\overline{\mathfrak{O}}$, close to $\mathfrak{O}$ and satisfying $\mathfrak{P}$, as well as the associated objects $\overline{\mathfrak{D}}_{i}$ to $\overline{\mathfrak{D}}$. We call approximate to an algorithm solving a problem of the above type. Here, the notion of "closeness" depends in general on the particular problem that one is solving.

One can find in the literature approximate algorithms for computing gcds (see Corless et al., 1995; Emiris et al., 1997; Pan, 1996), factoring polynomials (see Corless et al., 2001; Galligo and Rupprech, 2002; Pan, 2001; Sasaki, 2001), etc. For algebraic varieties there also exist approximate solutions: see Corless et al. (2000), Dokken (2001) for the implicitization problem, in Farouki and Rajan (1988) the numerical condition of implicitly given algebraic curves and surfaces has been analyzed, and see Bajaj and Royappa (2000), Gahleitner et al. (2002), Hartmann (2000), Pérez-Díaz et al. (2004, 2005) where the parametrization questions are treated.

In this paper we consider the approximate parametrization problem for affine plane algebraic curves. That is, with the above terminology, $\mathfrak{O}^{*}$ is an affine plane curve, $\mathfrak{P}$ is the fact of being rational, and $\mathfrak{D}_{i}^{*}$ is a rational parametrization of $\mathfrak{O}^{*}$. 
So, the problem is stated as follows: we are given an affine curve (say that it is a perturbation of a rational curve) and we want to compute a rational parametrization of a rational affine curve near it.

In Pérez-Díaz et al. $(2004,2005)$ the approximate parametrization problem is solved for the special case of affine plane curves and affine surfaces being a perturbation of a monomial curve and surface, respectively. In both papers, the basic tool is the use of $\epsilon$-points (see also Pérez-Díaz et al., 2006). More precisely, given a tolerance $\epsilon>0$, in Pérez-Díaz et al. (2004), the parametrization problem is solved for the case of affine plane curves having an $\epsilon$-singularity of maximum multiplicity, and in Pérez-Díaz et al. (2005) the problem is solved for affine surfaces having also an $\epsilon$-singularity of maximum multiplicity. The basic idea was to use a pencil of lines through the $\epsilon$-singularity and, hence, it was solved working as in the exact case for monomial varieties.

In this paper, we generalize the ideas of Pérez-Díaz et al. (2004) to the case of $d$-degree affine plane curves with $d$ different points at infinity. For this purpose, the first obstacle is to associate suitably the different $\epsilon$-singularities. This leads to the notion of cluster. Then, we introduce the notion of (affine) $\epsilon$-rationality, and we provide an algorithm to parametrize approximately $\epsilon$-rational. The idea of the algorithm is to work with linear systems of curves of degree $d-2$. This system plays the role of the linear system of adjoint curves in the exact parametrization algorithm. In addition, we prove that the degree of the output rational curve is the degree of the input one, and that both curves have the same points at infinity, and hence with the same real asymptotes.

This type of approximated problems is applicable by itself since it faces symbolic computation to real world problems. In addition, providing parametric representations of algebraic geometric objects helps with achieving computations and further manipulations of the object. This is of special interest in the field of CAGD. For instance when considering surfacesurface intersection or, in particular, when performing planar sections. In Example 5.3, for a given surface, we show how our algorithm detects planar intersections that, although are not rational, are $\epsilon$-rational. Therefore we provide rational parametrizations to deal with these (non-rational) planar curve intersections.

Associated to this type of problems appears, as a natural question, the closeness analysis between the input and output curves of the algorithm. In our case, this closeness notion is given by the Hausdorff distance (see Section 6). That is, we say that the input and output curves are close if their Haussdorf distance (as real curves) is small related to the tolerance. As we have stated above, both curves have the same points at infinity. This property, jointly with the hypothesis that the input curve has $d$ different points at infinity, ensures that the Hausdorff distance between them is finite (see lemma 6.1). In addition to the distance measure between input and output curves, one can go an step further and consider an additional question, namely whether the algorithm returns the best (in the sense of the closest to the input) solution. For instance, in our case, we can identify every projective plane curve, by means of its coefficients, with a point on a projective space. Then, for $d$ sufficiently big, the rational curves form a sub-variety $\mathcal{W}$ of this projective space. Therefore, one can consider the computation of an element in $\mathcal{W}$ minimizing the (Hausdorff) distance to the input curve. Unfortunately we have not been able to complete a theoretical analysis of the distance, nor on the minimization of the solution. We believe that, although interesting, both problems are very hard and we leave them as future research. Instead, we analyze two particular examples (a bounded and a non-bounded curve), where we describe a theoretical method to deal with the problem and where we estimate the distance. Every example we have tried shows that the curves are close, and it allows us to think about a theoretical treatment of this fact as a future project.

The рарег is structured as follows. In Section 1 we recall the main notions and properties on $\epsilon$-singularities. Section 2 is devoted to recall the main ideas of the exact parametrization algorithm for curves. In Section 3 we develop the idea of cluster and we introduce the notion of $\epsilon$-rationality. In Section 4 we derive the approximate algorithm, as well as the main properties of the output curve. In Section 5 we illustrate the algorithm through several examples, and in Section 6 we empirically analyze the errot.

Throughout this paper, we use the following terminology. $\|\cdot\|$ and $\|\cdot\|_{2}$ denote the polynomial $\infty$-norm and the usual unitary norm in $\mathbb{T}^{2}$, respectively. $\mid$. I denotes the module in $\mathbb{T}$. The partial derivatives of a polynomial $g \in \mathbb{C}[x, y]$ are denoted by $g^{\bar{y}}:=\frac{\hat{y}^{1+j} g}{y^{i} x^{j} y}$ where $\bar{v}=(i, j) \in \mathbb{N}^{2}$; we assume that $g^{\overrightarrow{0}}=g$. Moreover, for $\bar{v}=(i, j) \in \mathbb{N}^{2},|\bar{v}|=i+j$. Also, $\bar{e}_{1}=(1,0)$, and $\vec{e}_{2}=(0,1)$.

In addition, we use the following general assumptions. A tolerance $\epsilon$ is fixed such that $0<\epsilon<1$. $\mathcal{C}$ is an affine real plane algebraic curve over $\mathbb{C}$ of proper degree $d>0$ (see Definition 1.1), with $d$ different points at infinity, not passing through $(1: 0: 0),(0: 1: 0)$, and defined by an $\epsilon$-irreducible polynomial $f(x, y) \in \mathbb{R}[x, y]$; that is $f$ can not be expressed as $f(x, y)=g(x, y) h(x, y)+\mathcal{E}(x, y)$ where $h, g, \mathcal{E} \in \mathbb{C}[x, y]$ and $\|\mathcal{E}(x, y)\|<\epsilon\|f(x, y)\|$ (see Corless et al., 2001; Kaltofen et al., 2008). We denote by $\mathcal{C}^{h}$ the projective closure of $\mathcal{C}$. Let us mention that the condition $(1: 0: 0),(0: 1: 0) \notin \mathcal{C}^{h}$ can be avoided by performing a suitable affine orthogonal linear change of coordinates.

\section{Preliminaries on $\epsilon$-points}

Our fundamental technique to deal with the approximate parametrization problem is the use of $\epsilon$-points. The notion of $\epsilon$-point of an algebraic variety was introduced by the authors (see Pérez-Díaz et al., 2004, 2005, 2006) as a generalization of the notion of approximate root of a univariate polynomial. In this section, we briefly summarize some previous notions introduced in Pérez-Díaz et al. (2004, 2005), and geometric properties obtained in Pérez-Díaz et al. (2006). We start with the notion of proper degree. 
Definition 1.1. We say that a polynomial $g \in \mathbb{S}[x, y]$ has proper degree $\ell$ if the total degree of $g$ is $\ell$, and $\exists \vec{v} \in \Gamma^{2}$, with $|\bar{v}|=\ell$, such that $\left|g^{\vec{v}}\right|>\in\|g\|$.

We say that an algebraic plane curve has proper degree $\ell$ if its defining polynomial has proper degree $\ell$.

The notion of $\epsilon$-point is as follows.

Definition 1.2. $P \in \mathbb{C}^{2}$ is an $\epsilon$-(affine) point of $\mathcal{C}$ if $|f(P)|<\epsilon\|f\|$.

In this situation, we introduce the notion of $\epsilon$-singularity, pure $\epsilon$-singularity, and $\epsilon$-ramification point.

Definition 1.3. Let $P \in \mathbb{C}^{2}$ be an $\in$-point of $\mathcal{C}$ :

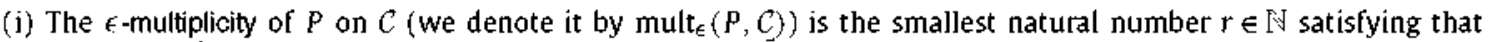

(1) $\forall \vec{v} \in \mathbb{N}^{2}$, such that $0 \leqslant|\vec{v}| \leqslant r-1$, it holds that $\left|f^{v_{\{}}(P)\right|<\in\|f\|$,

(2) $\exists \bar{v} \in \mathbb{N}^{2}$, with $|\vec{v}|=r$, such that $\left|f^{v_{1}}(P)\right| \geqslant \epsilon\|f\|$

(ii) $P$ is an $\epsilon$-(affine) simple point of $\mathcal{C}$ if mult $\epsilon(P, \mathcal{C})=1$; otherwise, $P$ is an $\epsilon$-(affine) singularity of $\mathcal{C}$.

(iii) $P$ is a $k$-pure $\epsilon$-singularity of $\mathcal{C}$, with $k \in\{1,2\}$, if $\operatorname{mult}_{\epsilon}(P, \mathcal{C})>1$ and $\mid f$ mult $_{\epsilon}\left(P, \mathcal{C}, \vec{e}_{k}(P) \mid \geqslant \epsilon\|f\|\right.$.

(iv) $P$ is an $\epsilon$-(affine) ramification point of $\mathcal{C}$ if mult $\epsilon(P, \mathcal{C})=1$, and either $\left|f^{\tilde{e}_{1}}(P)\right|<\epsilon\|f\|$ or $\left|f^{e_{2}}(P)\right|<\epsilon\|f\|$.

Note that, since $\mathcal{C}$ has proper degree, $0 \leqslant \operatorname{mult}(P, \mathcal{C}) \leqslant \operatorname{mult}_{\epsilon}(P, \mathcal{C}) \leqslant \operatorname{deg}(\mathcal{C})$, where mult $(P, \mathcal{C})$ denotes the "exact" multiplicity of $P$ on $\mathcal{C}$. For instance, the origin has exact multiplicity 1 , and $\epsilon$-multiplicity 2 , on the curve defined by $\frac{\epsilon}{2} x+x^{3}+y^{2}$. In the exact case, if $\mathcal{C}$ is irreducible, mult $(P, \mathcal{C})<\operatorname{deg}(\mathcal{C})$. Thus one may expect that in the approximate case, if $\mathcal{C}$ is $\epsilon$-irreducible, then $\operatorname{mult}_{\epsilon}(P, \mathcal{C})<\operatorname{deg}(\mathcal{C})$. Although this is the case in all the examples we have tried, we have not been

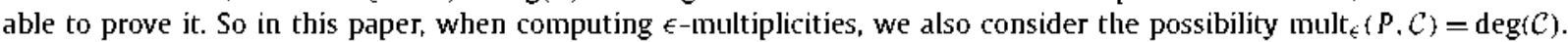

Definition 1.4. Let $P$ be an $\epsilon$-point of $\mathcal{C}$ and $r=$ mult $_{\epsilon}(P, \mathcal{C})$. If $P$ is $k$-pure, with $k \in\{1,2\}$, we define the $k$-weight of $P$ as

$$
\text { weight }_{k}(P)=\max _{i=0 \ldots, r-1}\left\{\left|\frac{r ! \cdot f^{i \cdot \vec{e}_{k}}(P)}{i ! \cdot f^{r \cdot \vec{e}_{k}}(P)}\right|^{\frac{1}{r-i}}\right\} \text {. }
$$

We define the weight of $P$, denoted by weight $(P)$, as $\max _{\{\text {weight }}(P)$, weight $\left.{ }_{2}(P)\right\}$, if $P$ is pure in both directions, and as the corresponding $k$-weight otherwise.

The following rational function, introduced in Sasaki and Terui (2002), will be used in this development: $\mathcal{R}_{\text {out }}(x)=$ $\frac{1}{2}-\frac{x(1-9 x)}{2(1+3 x)}-\frac{32 x^{2}}{(1+3 x)^{3}}$

\section{Preliminaries on symbolic parametrization}

In this section, we briefly recall the symbolic parametrization algorithm for rational plane algebraic curves of degree $d>2$ (note that lines and conics can be trivially parametrized by lines) based on ( $d-2$ ) adjoint curves; for further details see Sendra et al. (2007). For this purpose, throughout this section we assume that $\mathcal{C}$ is rational (i.e. its genus is zero). In addition, taking into account our requirements in Section 4 and for simplicity sake, we assume in this section that all singularities are affine and ordinary. Again, for a complete description see Sendra et al. (2007).

The idea is to use a linear system of curves such that for almost every curve in this system, all its intersections with $C^{\text {h }}$, except one, are predetermined; recall that $\mathcal{C}^{h}$ is the projective closure of $c$. Moreover, the set of all these intersection points is the same one for every curve in the system, and the points in this set are called the "base points". Thus, if one computes the intersection points of $\mathcal{C}^{h}$ with a generic representative of the system, the expression of the unknown intersection point gives the parametrization of the curve in terms of the parameter defining the linear system.

More precisely, let $\mathcal{H}_{d-2}$ be the linear system of adjoint curves to $\mathcal{C}^{\text {h }}$ of degree $d-2$. That is, $\mathcal{H}_{d-2}$ is the linear system of curves of degree $d-2$ having each $r$-fold of $c^{\text {h }}$ as a base point of multiplicity $r-1$; i.e, as a point of multiplicity at least $r-1$. In particular it implies that the multiplicity of intersection of a curve in $\mathcal{H}_{d-2}$ and $\mathcal{C}^{\text {h }}$ at a base point of multiplicity $r-1$ is at least $r(r-1)$. Thus, using that the genus of $\mathcal{C}$ is zero, and taking into account Bézout's Theorem, one deduces that $d-2$ intersections of $\mathcal{C}^{h}$ and a generic element in $\mathcal{H}_{t-2}$ are not predetermined. In this situation, one may take ( $\left.d-3\right)$ simple points on $\mathcal{C}^{h}$, and determine the 1-dimensional linear subsystem $\mathcal{H}_{d-2}^{*}$ of $\mathcal{H}_{t-2}$ obtained when these simple points are required to be base points of multiplicity 1. In this way, the number of predetermined intersections (counted with multiplicity $)$ is $(d-1)(d-2)+(d-3)$, i.e. only one intersection point is missing. Thus, computing this free intersection one finds a rational parametrization of $c^{h}$. Summarizing these ideas one has the following process:

(1) Compute the singularities of $\mathcal{C}^{h}$ as well as their multiplicities (recall that we have assumed that all singularities are affine and ordinary). 
(2) Determine the linear system $\mathcal{H}_{d-2}$ of adjoint curves of degree $(d-2)$ to $\mathcal{C}^{\text {h }}$.

(3) Compute d-3 different simple points on $\mathcal{C}^{h}$.

(4) Determine the linear subsystem $\mathcal{H}_{d-2}^{*}$ of $\mathcal{H}_{d-2}$ by requiring that every simple points in Step (3) is a base point of multiplicity one.

(5) Compute the free intersection point of $\mathcal{H}_{d-2}^{*}$ and $c^{\text {h }}$.

Let us make a comment on how to computationally perform the steps in the above process. Step (1) can be performed, for instance, using resultants. In Step (2), one considers a homogeneous polynomial $H(x, y, z)$ of degree $(d-2)$ with undetermined coefficients. Now, for each singular point $P$ of multiplicity $r$ one requires that $H$ and all its partial derivatives till order $(r-1)$ vanish at $P$. This generates a linear system of equations in the undetermined coefficients of $H$. Solving it, and substituting in $H$, we get the defining polynomial of $\mathcal{H}_{t-2}$; let us call it again $H$. Step (3) may be performed by intersecting $C^{h}$ with lines (see Sendra et al., 2007 for advanced approaches); although it is not necessary, looking for the parallelism with the reasoning in Section 4, we take affine simple points. Step (4) can be approached as Step (2), i.e. requiring that $H$ vanishes at each simple point, solving the provided linear system and substituting the solution in $H$; let $H^{*}(t, x, y, z)$ be the defining polynomial of $\mathcal{H}_{d-2}^{*}$ (note that $\operatorname{dim}\left(\mathcal{H}_{d-2}^{*}\right)=1$ ). Finally, let us deal with Step (5). For this purpose, let $\left\{Q_{i}:=\left(q_{i, 1}: q_{i, 2}: 1\right)\right\}_{i=1, \ldots, s}$ be the singularities and $r_{i}$ the multiplicity of $Q_{i}$. Also, let $\left\{P_{j}:=\left(p_{i, 1}: p_{i, 2}: 1\right)\right\}_{1, \ldots, t-3}$ be the simple points determined in Step (3). Then, the free intersection point is obtained by computing the primitive part, w.r.t. $t$, of the resultants of $H^{*}(t, x, y, 1)$ and $f(x, y)$ with respect to $x$ and $y$, respectively. Indeed, it holds that (see San Segundo and Sendra, 2005)

$$
\begin{aligned}
& S_{1}(x, t)=\operatorname{Res}_{y}\left(H^{*}(t, x, y, 1), f(x, y)\right)=\prod_{i=1}^{s}\left(x-q_{i, 1}\right)^{r_{i}\left(r_{j}-1\right)} \prod_{i=1}^{d-3}\left(x-p_{i, 1}\right) M_{1}(x, t), \\
& S_{2}(y, t)=\operatorname{Res}_{x}\left(H^{*}(t, x, y, 1), f(x, y)\right)=\prod_{i=1}^{s}\left(y-q_{i, 2}\right)^{r_{j}\left(r_{i}-1\right)} \prod_{i=1}^{d-3}\left(y-p_{i, 2}\right) M_{2}(y, t),
\end{aligned}
$$

where $\operatorname{deg}_{x}\left(M_{1}\right)=\operatorname{deg}_{y}\left(M_{2}\right)=1$. Therefore, the parametrization is the solution in $\{x, y\}$ of $\left\{M_{1}(x, t)=0, M_{2}(y, t)=0\right\}$.

\section{3. $€$-rational curves}

The main goal of this section is to provide an alternative definition of rational curve for the approximate frame; namely, the concept of $\epsilon$-rationality. This notion is related to the $\epsilon$-singularities and their $\epsilon$-multiplicity. As explained in the introduction, we assume that we are given a perturbation of a rational curve. For a given singularity of this rational curve the effect of the perturbation would be the "explosion" generating a set of $\epsilon$-singularities, with different $\epsilon$-multiplicities, of the curve we work with. Therefore, differently from the exact case, we will have in general more $\epsilon$-singularities than expected, and their nature indicates that they need to be associated; we will solve this difficulty introducing a suitable concept of cluster. For this purpose, we faced two main difficulties. On one hand, deciding how to associate $\epsilon$-singularities to give an appropriate definition of cluster and on the other computing the $\epsilon$-multiplicity of a cluster.

To determine the $\epsilon$-singularities, we compute numerically the set $\mathcal{S}_{T}$ of solutions of the algebraic system $T=$ $\left\{f^{\overrightarrow{0}}(x, y)=0, f^{\vec{e}_{1}}(x, y)=0, f^{\vec{e}_{2}}(x, y)=0\right\}$. Note that $f$ is irreducible, and hence $\mathcal{T}$ has finitely many solutions. Now, for each $P \in \mathcal{S}_{T}$, we check whether $\max \left\{\left|f^{\overrightarrow{0}}(P)\right|,\left|f^{\vec{e}_{1}}\{P\}\right|,\left|f^{\vec{e}_{2}}\{P)\right|\right\} \leqslant \epsilon\|f\|$. Let $\mathcal{S}_{1}$ be the subset of $\mathcal{S}_{T}$ satisfying this inequality. Given $P \in \mathcal{S}_{1}$, the computation of $\operatorname{mult}_{\epsilon}(P, \mathcal{C})$. can be obviously done by substituting $P$ at the corresponding partial derivatives and checking the conditions in Definition 1.3(1). However, for each $\epsilon$-point $P$ there exists an open disk centered at $P$ consisting of $\epsilon$-points of $\epsilon$-multiplicity at least mult $\epsilon(P, \mathcal{C})$ (see Lemma 3 in Pérez-Diaz et al., 2004). So, an small perturbation of $P$ may produce a different answer for the $\epsilon$-multiplicity. We are indeed interested in assigning the maximum possible $\epsilon$-multiplicity to the $\epsilon$-points we associate in a cluster (as will be defined later). The proof of Lemma 3 in Pérez-Díaz et al. (2004) shows how to detect the radius of one of these open disks, so one may try to estimate the maximum $\epsilon$-multiplicity at the disk. Nevertheless, in practice, this is unfeasible. Instead, we propose a different strategy that for practical purposes increases the chances of assigning the appropriate $\epsilon$-multiplicity to a defined cluster. Unfortunately it does not ensure the achievement of the maximum $\epsilon$-multiplicity on the set of $\epsilon$-singularities obtained.

More precisely, for each $k \in\{2 \ldots, d-1\}$, we take $\vec{u}_{1}, \ldots, \vec{u}_{s} \in \mathbb{S}^{2}$, with $2 \leqslant s \leqslant k+1$ (in practice $s=2$ ) such that for all $i,\left|\bar{u}_{i}\right|=k$ and $\operatorname{gcd}\left(f^{\vec{u}_{1}}, \ldots, f^{\vec{u}_{s}}\right)=1$, and we solve numerically $\left\{f^{\vec{u}_{1}}=0, \ldots, f^{\vec{u}_{s}}=0\right\}$. Let $\mathcal{A}_{k}$ be the set of solutions. Then, for $k \in\{2, \ldots, d-1\}$ we consider the set (note that $\mathcal{S}_{1}$ is defined above)

$$
\mathcal{S}_{k}=\left\{P \in \mathcal{A}_{k} /\left|f^{\bar{w}}(P)\right|<\epsilon\|f\| \forall \bar{w} \in \mathbb{N}^{2} \text { with }|\bar{w}| \leqslant k\right\} .
$$

If for a given $k$ and for all $s$ it holds that $\operatorname{gcd}\left(f^{\vec{u}_{1}}, \ldots, f^{\vec{u}_{s}}\right) \neq 1$, we take $\mathcal{S}_{k}=\emptyset$. Finally we consider the set

$$
\mathcal{S}=\bigcup_{k=1}^{d-1} \mathcal{S}_{k}
$$


We explain next how to identify the $\epsilon$-singularities in $\mathcal{S}$, the cluster construction that will lead us to the concept of $\epsilon$ rationality. In the identification process, having $\epsilon$-singularities in $\mathcal{S}_{k}$ for higher values of $k$, will increase the chances of detecting the $\epsilon$-multiplicity of the cluster.

Definition 3.1. The set $\mathcal{S}$, introduced above, is called the $\epsilon$-(affine)-singular locus of $\mathcal{C}$. We denote it by $\operatorname{Sing} \epsilon(\mathcal{C})$.

Example 3.2. Let $\epsilon=0.001$ and let $\mathcal{C}$ be defined by $f(x, y)=x^{3} y+y^{3} x+x^{3}+\frac{\epsilon}{2} x^{2}+\epsilon y+\frac{\epsilon}{2}$. Then $\operatorname{Sing}_{\epsilon}(\mathcal{C})=\mathcal{S}_{1} \cup \mathcal{S}_{2} \cup \mathcal{S}_{3}$ where

$$
\begin{aligned}
\mathcal{S}_{1}=\left\{P_{1}\right. & =(0.02131893405+0.009609927603 i, 0.02442855631+0.1171004584 i), \\
P_{2} & =(0.004713033954+0.02355323617 i,-0.07491796596-0.09032199938 i), \\
P_{3} & =(-0.01424770212+0.01818884517 i, 0.1084633939+0.05315246871 i), \\
P_{4} & =(-0.02443272919,-0.1159479025), \\
P_{5} & =(-0.01424770212-0.01818884517 i, 0.1084633939-0.05315246871 i), \\
P_{6} & =(0.004713033954-0.02355323617 i,-0.07491796596+0.09032199938 i), \\
& \left.P_{7}=(0.02131893405-0.009609927603 i, 0.02442855631-0.1171004584 i)\right\}, \\
S_{2}=\left\{P_{8}\right. & =(-0.0001666666667,0)\} . \\
S_{3}= &
\end{aligned}
$$

Moreover, $\operatorname{mult}_{\epsilon}\left(P_{1}\right)=\cdots=$ mult $_{\epsilon}\left(P_{7}\right)=2$ but $\operatorname{mult}_{\epsilon}\left(P_{8}\right)=3$. Note that considering only $\mathcal{S}_{1}$ we would have not found a point with $\epsilon$-multiplicity 3.

As we could check in the previous example, the difficulty appears when two (in general more than two) $\epsilon$-singularities $P$ and $Q$ are very "close", because somehow we need to identify them. To approach this, first we assign a radius to each $\epsilon$-singularity (see Definition 3.3); i.e. the $\epsilon$-singularity is seen as a closed Euclidean disk. Secondly we associate the disks, by means of an equivalence relation (see Definition 3.5), yielding the singularity clusters (see Definition 3.6). Finally we choose, among the finitely many points in the cluster, a representative that maximizes the $\epsilon$-multiplicity and minimizes in module the evaluation of $f$ at the point (see Definition 3.6).

We start with the notion of radius, which is motivated by its good performance in practice.

Definition 3.3. Let $P$ be an $\epsilon$-point of $\mathcal{C}$. We define its radius, and we denote it by radius $(P)$, as $\mathcal{R}_{\text {out }}$ (weight $\left.(P)\right)$ if $P$ is pure and zero otherwise.

Definition 3.4. Let $\mathcal{A}$ be a finite set of $\epsilon$-points of $\mathcal{C}$. We define on $\mathcal{A}$ the following binary relation: for $P, Q \in \mathcal{A}$, we say that

$$
P \mathcal{R}^{*} Q \Longleftrightarrow\|P-Q\|_{2}+|\operatorname{radius}(P)-\operatorname{radius}(Q)|<\mathcal{R}_{\text {out }}(\epsilon)
$$

$\mathcal{R}^{*}$ is reflexive and symmetric but it is not in general transitive. In order to have an equivalence relation, we consider its transitive closure.

Definition 3.5. Let $\mathcal{A}$ be a finite set of $\epsilon$-points of $\mathcal{C}$. We define on $\mathcal{A}$ the following equivalence relation: for $P, Q \in \mathcal{A}$, we say that

$$
P \mathcal{R Q} \Longleftrightarrow\left\{\begin{array}{l}
P \mathcal{R}^{*} Q \\
\text { or there exist } P_{1}, \ldots, P_{n} \in \mathcal{A} \text { such that } P \mathcal{R}^{*} P_{1}, \ldots, P_{n} \mathcal{R}^{*} Q
\end{array}\right.
$$

Definition 3.6. Let $\mathcal{A}$ be a finite set of $\epsilon$-points of $\mathcal{C}$. For $P \in \mathcal{A}$ we define the cluster of $P$ w.r.t. $\mathcal{A}$ as its equivalence class under $\mathcal{R}$.

We say that $R$ is a (canonical) representative of a cluster $\mathfrak{C}$ if: $R \in \mathfrak{C}$, for all $R^{\prime} \in \mathfrak{C}$ it holds that $\operatorname{mult}_{\epsilon}(R, \mathcal{C}) \geqslant$ mult $_{\epsilon}\left(R^{\prime}, \mathcal{C}\right)$, and for all $R^{\prime} \in \mathcal{C}$ such that mult $\operatorname{mon}_{\epsilon}(R, \mathcal{C})=$ mult $_{\epsilon}\left(R^{\prime}, \mathcal{C}\right)$ it holds that $|f(R)| \leqslant\left|f\left(R^{\prime}\right)\right|$.

We define the $\epsilon$-multiplicity of the cluster as the $\epsilon$-multiplicity of any of its canonical representatives.

We denote a cluster by $\operatorname{Cluster}_{r}(R, \mathcal{A})$, where $r$ is the $\epsilon$-multiplicity and $R$ a canonical representative, and by $\operatorname{Cluster}_{r}(R)$ when $\mathcal{A}=\operatorname{Sing}_{\epsilon}(\mathcal{C})$ 
The notion of cluster is based on the equivalence relation $\mathcal{R}$, that is constructed from $\mathcal{R}^{*}$. In order to motivate $\mathcal{R}^{*}$, take into account that two $\epsilon$-singularities are associated if their disks are a small vibration of each other. This might be because the centers, or the radios, or both, are a small perturbation of each other. These phenomena are controlled in the definition of $\mathcal{R}^{*}$; the first summand in Definition 3.4 measures the vibration of the centers and the second does it for the radios.

Finally, when we introduce the notion of canonical representative the first requirement is about the multiplicity, while the second is about the value of the implicit equation at the point. With this strategy we try to increase the possibilities of achieving $\epsilon$-genus zero (see Definition 3.7). Of course, one might consider the contrary criterion (i.e. first the module and second the multiplicity). Nevertheless, we do not provide a theoretical analysis of validation for our particular criterion election, but in all our examples the results were satisfactory.

Now, we are ready to introduce the notion of $\epsilon$-rationality.

Definition 3.7. If $\left\{\text { Ciuster }_{\mathrm{r}_{j}}\left(P_{\mathrm{i}}\right)\right\}_{i=1, \ldots, s}$ is the cluster decomposition of $\operatorname{Sing}_{\epsilon}(\mathcal{C})$, we say that $\mathcal{C}$ is $\epsilon$-(affine) rational if $(d-1)(d-2)-\sum_{i=1}^{s} r_{i}\left(r_{j}-1\right)=0$.

Remark 3.8. Note that in the previous theoretical development we have not considered singularities (neither $\epsilon$-singularities) at infinity. We leave this extension of the concept of $\epsilon$-rationality for further research.

If we apply the previous ideas to Example 3.2, with $\epsilon=0.001$, we get that the 8 points of Sing $(\mathcal{C})$ belong to the same cluster. So, the cluster decomposition is $\left\{\right.$ Ciuster $\left._{3}\left(P_{8}\right)=\left\{P_{1}, \ldots, P_{8}\right\}\right\}$. Therefore, $C$ is $\epsilon$-rational; indeed, it is $\epsilon$-monomial, and thus parametrizable with the techniques in Pérez-Díaz et al. (2004). We finish the section with a more general example.

Example 3.9. Let us consider $\epsilon=0.005$ and the curve $\mathcal{C}$ of proper degree 5 defined by the polynomial (see Fig. 1):

$$
\begin{aligned}
f(x, y)= & -\frac{106029}{48200} x^{2}-\frac{10593}{48200} x^{4} y-\frac{43461}{48200} x^{3} y^{2}+\frac{17919}{9640} x^{3}-\frac{91179}{48200} y^{4}+\frac{99}{100} x y^{3}+\frac{99}{100} x^{2} y \\
& -\frac{25443}{24100} x^{2} y^{3}+\frac{8217}{24100} y^{2}+\frac{99}{100} x^{4}+\frac{99}{100} x y^{4}+\frac{99}{100} y^{3}-\frac{9009}{48200} x^{5}+\frac{252351}{48200} x y^{2}-\frac{42669}{24100} x^{2} y^{2} \\
& +\frac{69993}{48200} x^{3} y-\frac{6943}{48200} x y-\frac{25443}{48200} y^{5}+\frac{1}{100} .
\end{aligned}
$$

The $\epsilon$-singular locus is $\operatorname{Sing}_{\epsilon}(\mathcal{C})=\mathcal{S}_{1} \cup \mathcal{S}_{2} \cup \mathcal{S}_{3}$, where

$$
\begin{aligned}
& \mathcal{S}_{1}=\left\{P_{1}\right.=(-0.9956027274+0.0004067223817 i, 0.001447687187+0.9982777543 i), \\
& P_{2}=(1.011706789-0.1320874194 i,-1.008532436+0.06832949372 i), \\
& P_{3}=(1.007458642,-1.044045331), P_{4}=(0.9909273695,-0.9540334161), \\
& P_{5}=(1.011706789+0.1320874194 i,-1.008532436-0.06832949372 i), \\
& P_{6}=(-0.9956027274-0.0004067223817 i, 0.001447687187-0.9982777543 i), \\
&\left.P_{7}=(0,0)\right\}, \\
& S_{2}=\left\{P_{8}=(1.000000001,-1 .)\right\}, \\
& S_{3}=
\end{aligned}
$$

Moreover, mult $_{\epsilon}\left(P_{1}\right)=$ mult $_{\epsilon}\left(P_{2}\right)=$ mult $_{\epsilon}\left(P_{7}\right)=2$, and mult $_{\epsilon}\left(P_{3}\right)=$ mult $_{\epsilon}\left(P_{4}\right)=$ mult $_{\epsilon}\left(P_{5}\right)=$ mult $_{\epsilon}\left(P_{6}\right)=$ mult $_{\epsilon}\left(P_{8}\right)=3$. Furthermore, the cluster decomposition is (see Fig. 1):

$$
\begin{aligned}
& \text { Eltister }_{2}\left(\mathrm{P}_{1}\right)=\left\{P_{1}\right\}, \\
& \text { Eltister }_{2}\left(\mathrm{P}_{2}\right)=\left\{P_{2}\right\}, \\
& \text { Eltuster }_{2}\left(\mathrm{P}_{7}\right)=\left\{P_{7}\right\}, \\
& \text { Cluster }_{3}\left(\mathrm{P}_{8}\right)=\left\{P_{3}, \mathrm{P}_{4}, \mathrm{P}_{5}, P_{6}, P_{8}\right\} .
\end{aligned}
$$

Thus, $C$ is $\epsilon$-rational.

\section{Approximate parametrization algorithm}

In this section, we present our approximate parametrization algorithm. For this purpose, we assume that $\mathcal{C}$ is $\epsilon-$ rational of proper degree $d>2$ (note that for $d=1$ the problem is trivial, and for $d=2$ one can apply the algorithm by Pérez-Díaz et al, 2004), and that

$$
\left\{\text { Clister }_{r_{i}}\left(Q_{i}\right)\right\}_{i=1 \ldots . . . s} \text {, where } Q_{i}:=\left(q_{j, 1}: q_{i, 2}: 1\right)
$$



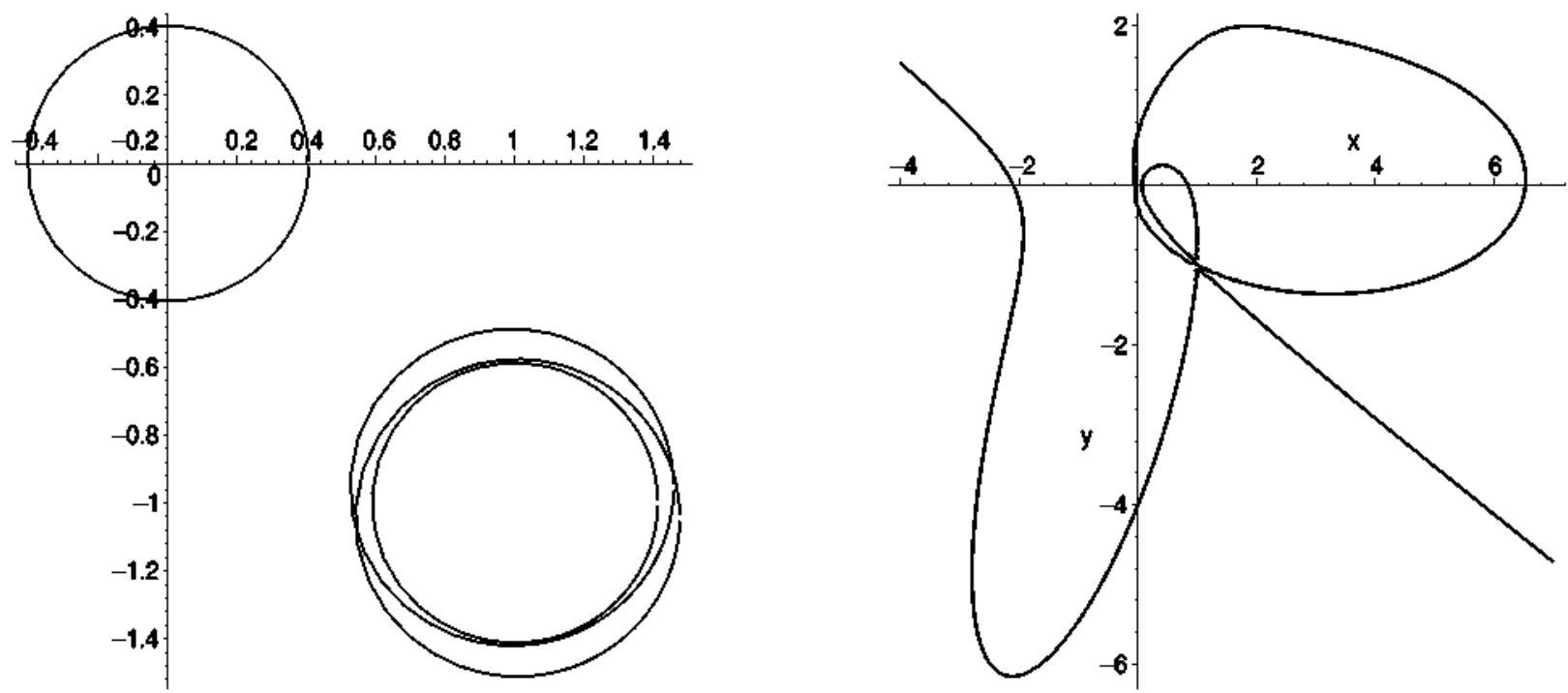

Fig. 1. Left: Clusters, Right; Curve $C$.

is the cluster decomposition of Sing $(\mathcal{C})$. Furthermore, if possible, i.e. when there exists a real canonical representative of the cluster, we take $Q_{i}$ real.

In this situation, the strategy is to adapt the algorithm in Section 2 as follows. Let $\mathcal{C}^{\text {h }}$ be the projective closure of $\mathcal{C}$. We consider the linear system of curves $\overline{\mathcal{H}}_{d-2}$ of degree $(d-2)$ given by the divisor $\sum_{i=1}^{s} r_{j} Q_{i}$. That is, $Q_{i}$ is a base point of (exact) multiplicity $r_{i}-1$ of the linear system. Afterwards, one computes $(d-3) \epsilon$-simple affine points on $\mathcal{C}^{h}$ (see below for details), and determines the linear subsystem $\overline{\mathcal{H}}_{d-2}^{*}$ of $\overline{\mathcal{H}}_{d-2}$ obtained by intersecting $\overline{\mathcal{H}}_{d-2}$ with the linear system of $(d-2)$-degree curves generated by the divisor $\sum_{i=1}^{d-3} P_{i}$; say that $P_{i}:=\left(p_{i, 1}: p_{i, 2}: 1\right)$. If $P_{i}, Q_{j}$ would be exact points and singularities, respectively, of $\mathcal{C}^{h}$, then $\operatorname{dim}\left(\bar{H}_{t-2}^{*}\right)=1$ (see Chapter 4 in Sendra et al., 2007). However, in our case, since we are working with $\epsilon$-points we can only ensure that $\operatorname{dim}\left(\overline{\mathcal{H}}_{d-2}^{*}\right) \geqslant 1$ (see Theorem 2.56 in Sendra et al., 2007). If this dimension is strictly bigger than 1 , we can either take more $\epsilon$-simple points till dimension 1 is reached, or we can take an small perturbation of the $\epsilon$-points such that the effective divisor $\sum_{i=1}^{s} r_{i} Q_{i}+\sum_{i=1}^{d-3} P_{j}$ is in general position (see p. 49 in Sendra et al., 2007), and hence the dimension is 1 . So, we can assume w.l.o.g. that $\operatorname{dim}\left(\overline{\mathcal{H}}_{d-2}^{*}\right)=1$. Let, then, $H^{*}(t, x, y, z)$ be the defining homogeneous polynomial of $\overline{\mathcal{H}}_{d-2}^{*}$.

At this point, if $P_{i}, Q_{j}$ would be exact points and singularities, respectively, of $\mathcal{C}$, the symbolic algorithm presented in Section 2 would output the parametrization $\mathcal{P}_{(t)}=\left(\frac{p_{1}(t)}{q_{1}(t)}, \frac{p_{2}(t)}{q_{2}(t)}\right)$, where

$$
\begin{aligned}
& q_{1}(t) x-p_{1}(t)=\frac{\operatorname{Res}_{y}\left(H^{*}(t, x, y, 1), f(x, y)\right)}{\prod_{i=1}^{s}\left(x-q_{i, 1}\right)^{r_{j}\left(r_{i}-1\right)} \prod_{i=1}^{d-3}\left(x-p_{i, 1}\right)}, \\
& q_{2}(t) y-p_{2}(t)=\frac{\operatorname{Res}_{x}\left(H^{*}(t, x, y, 1), f(x, y)\right)}{\prod_{i=1}^{s}\left(y-q_{i, 2}\right)^{r_{j}\left(r_{i}-1\right)} \prod_{i=1}^{d-3}\left(y-p_{i, 2}\right)} .
\end{aligned}
$$

However, in our case, $P_{i}, Q_{j}$ are not exact points, but $\epsilon$-points. So these rational functions are not, in general, polynomials. Nevertheless, considering if necessary a small perturbation of $H^{*}$, the quotient of the division of each numerator by its denominator is linear as polynomial in either $x$ or $y$. Then, the idea is to determine the parametrization from these linear quotients. For this purpose, one may need to perform two perturbations, both affecting $H^{*}$. The first one ensures that the degree in the resultants is the expected one, namely $d(d-2)$, and hence it controls the degree of the output curve. The second guarantees that the output is indeed a parametrization; i.e. that not both components are constants. Note that, in the exact case, these two facts are provided by the theory. In this paper, we deal with the first perturbation leaving as degenerated cases those curves requiring the second perturbation. In Pérez-Díaz et al. (2009), one can see a complete analysis of the second degeneration.

More precisely, let $H^{*}(t, x, y, z)=H_{1}(x, y, z)+t H_{2}(x, y, z)$, and let $\mathcal{D}_{i}$ be the projective curve defined by $H_{i}, i=1,2$. We recall that $(1: 0: 0),(0: 1: 0) \notin \mathcal{C}^{h}$. Now, we need to ensure that either $\mathcal{C}^{h}, \mathcal{D}_{1}$ or $\mathcal{C}^{h}, \mathcal{D}_{2}$ do not have common points at infinity. If this is not the case, let $\left\{R_{1}, \ldots R_{m}\right\}$ be the points of $\mathcal{C}$ at infinity and $K\left(\rho_{1}, \rho_{2}, x, y, z\right)=\rho_{1} x^{d-2}+\rho_{2} y^{(t-2}$, where $\rho_{\mathrm{i}}$ are parameters. Then, we consider in $\mathbb{C}^{2}$ the union $\mathcal{L}$ of the affine lines defined by $H_{2}\left(R_{i}\right)+K\left(\rho_{1}, \rho_{2}, R_{i}\right)=0$, for $i=1, \ldots, m$. Note that, since $R_{i}$ are points at infinity, the polynomials $H_{2}\left(R_{i}\right)+K\left(\rho_{1}, \rho_{2}, R_{i}\right) \in \mathbb{C}\left[\rho_{1}, \rho_{2}\right]$ are not constant, and hence define lines. So, taking values for $\rho_{1}, \rho_{2}$ (say, small real numbers) we consider an small perturbation that ensures 
that the above requirement is satisfied. Thus, in what follows we assume that $\mathcal{D}_{2}$ and $\mathcal{C}^{\text {h }}$ do not have common points at infinity. Therefore, if $F$ is the homogenization of $f$, by Lemma 3.1 in Alcázar and Sendra (2005), one has that

$$
\operatorname{deg}_{x}\left(\operatorname{Res}_{y}\left(H^{*}, F\right)\right)=\operatorname{deg}_{y}\left(\operatorname{Res}_{x}\left(H^{*}, F\right)\right)=d(d-2) .
$$

Moreover, since $H^{*}$ and $\mathcal{C}^{h}$ do not have common points at infinity, it holds that

$$
\operatorname{deg}_{x}\left(\operatorname{Res}_{y}\left(H^{*}(t, x, y, 1), f\right)\right)=\operatorname{deg}_{y}\left(\operatorname{Res}_{x}\left(H^{*}(t, x, y, 1), f\right)\right)=d(d-2) .
$$

Now, we consider the polynomials

$$
A_{1}(x)=\prod_{i=1}^{s}\left(x-q_{i, 1}\right)^{r_{i}\left(r_{i}-1\right)} \prod_{i=1}^{d-3}\left(x-p_{i, 1}\right), A_{2}(y)=\prod_{i=1}^{s}\left(y-q_{i, 2}\right)^{r_{i}\left(r_{i}-1\right)} \prod_{i=1}^{d-3}\left(y-p_{i .2}\right) .
$$

Since $\mathcal{C}$ is $\epsilon$-rational, it holds that

$$
\operatorname{deg}_{x}\left(A_{1}(x)\right)=\operatorname{deg}_{y}\left(A_{2}(y)\right)=d(d-2)-1 .
$$

Let $B_{1}(x, t):=\bar{q}_{1}(t) x-\bar{p}_{1}(t)$ be the quotient of $S_{1}(x, t):=\operatorname{Res}_{y}\left(H^{*}(t, x, y, 1), f(x, y)\right)$ and $A_{1}(x)$. Similarly let $B_{2}(y, t):=$ $\bar{q}_{2}(t) x-\bar{p}_{2}(t)$ be the quotient of $S_{2}(y, t):=\operatorname{Res}_{x}\left(H^{*}(t, x, y, 1), f(x, y)\right)$ and $A_{2}(y)$. Then, we output

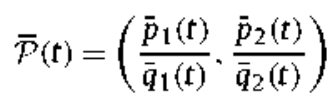

as approximate parametrization of $C$.

Before outlining the algorithm, we briefly describe how to proceed with the selection and computation of the (affine simple) $\epsilon$-points $P_{i}$. We first observe that, in general, an $\epsilon$-point can be computed by solving $\{f(x, y)=0, \alpha x+\beta y=\rho\}$, where $\alpha, \beta, \rho \in \mathbb{C}$. However, we are intersected in working with either real $\epsilon$-points or pairs of conjugate complex points. We can always compute all points, but at most one, in pairs of conjugate complex points. For choosing real points one can always analyze the roots of the discriminant of $f$ (see Theorem 7.7 in Sendra et al., 2007). On the other hand we have observed, in our examples, that taking (when possible) the simple $\epsilon$-points as (affine) $\epsilon$-ramification points (see Definition 1.3) the error distance between the original curve and the output curve decreases. So we tend to use first such points. Finally, one has to take care of the fact that a chosen $\epsilon$-point can be too close (i.e. in the same cluster) to an $\epsilon$-singularity or to a previously computed $\epsilon$-point, and hence identifiable with it. To avoid this, whenever a new simple $\epsilon$-point is computed we check whether it belongs to the cluster of the others points.

The above process provides the following approximate parametrization algorithm for deciding whether a real $\epsilon$-irreducible (with proper degree) plane algebraic curve $\mathcal{C}$ is $\epsilon$-rational, and in the affirmative case, compute an approximate parametrization. Recall that we assume that $\mathcal{C}$ has $d$ different points at infinity, and that $(0: 1: 0),(1: 0: 0) \notin \mathcal{C}^{h}$. If this last condition fails, one may consider an affine orthogonal change of coordinates to achieve the requirement.

\section{Approximate Parametrization Algorithm}

- Given a tolerance $\epsilon>0$ and an $\epsilon$-irreducible polynomial $f(x, y) \in \mathbb{0}[x, y]$, of proper degree $d>2$ (for $d=1$ it is trivial, if $d=2$ apply Pérez-Diaz et al., 2004), with $d$ different points at infinity, not passing through $(0: 1: 0),(1: 0: 0)$, and defining a real plane algebraic curve $\mathcal{C}$; let $F(x, y, z)$ be the homogenization of $f$.

- Decide whether $\mathcal{C}$ is $\epsilon$-rational and in the affirmative case.

- Compute a rational parametrization $\overline{\mathcal{P}}(t)$ of a curve $\bar{C}$.

(1) Compute the cluster decomposition $\left\{\mathcal{C}_{\text {hister }}\left(Q_{i}\right)\right\}_{i=1, \ldots, s}$ of $\operatorname{Sing}_{\epsilon}(\mathcal{C})$; say $Q_{i}=\left(q_{i, 1}: q_{i, 2}: 1\right)$.

(2) If $\sum_{i=1}^{s} r_{i}\left(r_{i}-1\right) \neq(d-1)(d-2)$. RETURN " $\mathcal{C}$ is not (affine) $\epsilon$-rational". If $s=1$ one may apply the algorithm in PérezDíaz et al. (2004).

(3) Determine the linear system $\overline{\mathcal{H}}_{t-2}$ of degree $(d-2)$ given by the divisor $\sum_{i=1}^{s} r_{j} Q_{i}$.

(4) Compute $(d-3) \epsilon$-ramification points $\left\{P_{j}\right\}_{1 \leqslant j \leqslant t-3}$ of $\mathcal{C}$; if there are not enough $\epsilon$-ramification points, complete with simple $\epsilon$-point. Take the points over $\mathbb{R}$, or as conjugate complex points. After each point computation check that it is not in the cluster of the others (including the clusters of $\left.Q_{i}\right) ;$ if this fails take a new one. Say $P_{i}=\left(p_{i .1}: p_{i .2}: 1\right)$.

(5) Determine the linear subsystem $\overline{\mathcal{H}}_{d-2}^{*}$ of $\overline{\mathcal{H}}_{d-2}$ given by the divisor $\sum_{i=1}^{d-3} P_{i}$. Let $H^{*}(t, x, y, z)=H_{1}(x, y, z)+$ $\mathrm{tH}_{2}(x, y, z)$ be its defining polynomial.

(6) If $\left[\operatorname{gcd}\left(F(x, y, 0), H_{1}(x, y, 0)\right) \neq 1\right]$ and $\left[\operatorname{gcd}\left(F(x, y, 0), H_{2}(x, y, 0)\right) \neq 1\right]$ replace $H_{2}$ by $H_{2}+\rho_{1} x^{d-2}+\rho_{2} y^{d-2}$, where $\rho_{1}, \rho_{2}$ are real and strictly smaller than $\epsilon$. Say that $\operatorname{gcd}\left(F(x, y, 0), H_{2}(x, y, 0)\right)=1$; similarly in the other case.

(7) $S_{1}(x, t)=\operatorname{Res}_{y}\left(H^{*}(x, y, 1), f\right)$ and $S_{2}(y, t)=\operatorname{Res}_{x}\left(H^{*}(x, y, 1), f\right)$.

(8) $A_{1}=\prod_{i=1}^{s}\left(x-q_{i, 1}\right)^{r_{i}\left(r_{i}-1\right)} \prod_{i=1}^{d-3}\left(x-p_{i, 1}\right), A_{2}=\prod_{i=1}^{s}\left(y-q_{i, 2}\right)^{r_{i}\left(r_{i}-1\right)} \prod_{i=1}^{d-3}\left(y-p_{i, 2}\right)$.

(9) For $i=1,2$ compute the quotient $B_{i}$ of $S_{i}$ by $A_{i}$ w.r.t. either $x$ or $y$.

(10) If the content of $B_{1}$ w.r.t. $x$ or the content of $B_{2}$ w.r.t. $y$ does depend on $t$, RETURN "degenerate case" (see Pérez-Díaz et al., 2009). 
(11) Determine the root $\bar{p}_{1}(t)$ of $B_{1}$, as a polynomial in $x$, and the root $\bar{p}_{2}(t)$ of $B_{2}$, as a polynomial in $y$.

(12) RETURN $\overline{\mathcal{P}}_{(t)}=\left(\overline{\mathrm{p}}_{1}(t), \bar{p}_{2}(t)\right)$.

\section{Remark 4.1 (General renuarks on the algorithm).}

1. It should be noted that the algorithm works symbolically with the exceptions of Steps 1 and 4 , where the $\epsilon$-singularities and $\epsilon$-simple points are determined. These points are computed numerically. Nevertheless, in order to be used in Steps 3 and 5 , they are converted to rational arithmetic.

2. Since the algorithm follows the steps of the exact approach, by Theorem 3.10 in Mnuk et al. (1997), one deduces that the worst case complexity is polynomial in the degree of the input curve.

3. In Step 9 of the algorithm, we only consider the quotients of the divisions, and we ignore the remainders. This remainders might be used to control the distance between the input and the output as shown in Corollary 6.4; note that, because of the construction, the polynomial $f$ evaluated at the parametrization is in fact the remainder. We leave such an study for future research.

4. Note that not every set of $(d-1)(d-2) / 2$ points, counted with multiplicity, is the singular locus of a rational curve of degree $d$. Nevertheless in our case, because of Lemma 3 in Pérez-Díaz et al. (2004), the singular divisor used in Step 3 of the algorithm can always be slightly perturbed so that it corresponds to a rational curve.

In the last part of this section, we state the main properties of the curve output by the algorithm. But first, we need some lemmas.

Lemma 4.2. The leading coefficient of $B_{1}(x, t)$ and $B_{2}(y, t)$ w.rt. $x$ and $y$, respectively (see Step 9 of the algorithm), are the same up to multiplication by non-zero constants in $C$. Furthermore, the roots are $\left\{-H_{1}(a, b, 0) / H_{2}(a, b, 0)\right\}_{(a t b)}\left(C^{\text {h }}\right.$ (see Step 5 of the aigoritlun).

Proof. Let $B_{1}(x, t)=q_{1}(t) x-p_{1}(t)$, and $B_{2}(y, t)=q_{2}(t) y-p_{2}(t)$. By hypothesis $F(1,0,0) \neq 0, F(0,1,0) \neq 0$. So, the leading coefficient of $F$ w.r.t. $y$ is a non-zero constant; similarly w.r.t. $x$. Thus, by well known properties on resultants (see, e.g. Lemma 4.3.1. by Winkler, 1996), it holds that up to multiplication by a non-zero element in $\mathbb{C}$ :

$$
\operatorname{Res}_{y}\left(H^{*}(t, x, y, 0), F(x, y, 0)\right)=S_{1}^{H}(x, 0, t), \quad \operatorname{Res}_{x}\left(H^{*}(t, x, y, 0), F(x, y, 0)\right)=S_{2}^{H}(y, 0, t) .
$$

where $H^{*}$ is as in Step 5 of the algorithm, and $S_{i}^{H}$ denotes the homogenization of

$$
S_{1}(x, t)=\operatorname{Res}_{y}\left(H^{*}(t, x, y, 1), f\right), \quad S_{2}(y, t)=\operatorname{Res}_{x}\left(H^{*}(t, x, y, 1), f\right) .
$$

Now, observe that $S_{1}^{H}(x, 0, t)=q_{1}(t) x^{d(t-2)}$, and $S_{2}^{H}(y, 0, t)=q_{2}(t) y^{(t(d-2)}$. Moreover, let $F(x, y, 0)$ factor as $F(x, y, 0)=$ $\prod_{i=1}^{d}\left(\beta_{i} x-\alpha_{i} y\right)$. Since $F(0,1,0) \neq 0$ then $\alpha_{j} \neq 0$ for all $i$. Hence, up to multiplication by non-zero constants

$$
\operatorname{Res}_{y}\left(H^{*}(t, x, y, 0), F(x, y, 0)\right)=\prod_{i=1}^{d} \operatorname{Res}_{y}\left(H^{*}(t, x, y, 0), \beta_{i} x-\alpha_{i} y\right)=(-1)^{d(d-2)} x^{d(d-2)} \prod_{i=1}^{d} H^{*}\left(t, \alpha_{i}, \beta_{i}, 0\right) \text {. }
$$

Analogously, $\operatorname{Res}_{x}\left(H^{*}(t, x, y, 0), F(x, y, 0)\right)=(-1)^{d(d-2)} y^{d(d-2)} \prod_{j=1}^{d} H^{*}\left(t, \alpha_{j}, \beta_{i}, 0\right)$. So, up to multiplication by non-zero constants $q_{1}(t)=q_{2}(t)=\prod_{i=1}^{t} H^{*}\left(t, \alpha_{i}, \beta_{i}, 0\right)=\prod_{i=1}^{t}\left(H_{1}\left(\alpha_{i}, \beta_{i}, 0\right)+t H_{2}\left(\alpha_{i}, \beta_{i}, 0\right)\right)$.

Lemma 4.3. $\operatorname{deg}_{t}\left(B_{1}(x, t)\right)=d$ and $\operatorname{deg}_{t}\left(B_{2}(y, t)\right)=d$.

Proof. First note that $\operatorname{deg}_{i}\left(B_{1}\right) \leqslant d$ and $\operatorname{deg}_{i}\left(B_{2}\right) \leqslant d$. The equality follows from the last equality in the proof of Lemma 4.2 , and using that $\mathrm{H}_{2}\left(\alpha_{i}, \beta_{i}, 0\right) \neq 0$ for all $i$.

Lemma 4.4. Let $\mathbb{L}$ be the algebraic closure of $\mathbb{C}(t)$, and $c_{1}, c_{2}$ two plane projective curves over $\mathbb{L}$ witl defining polynonials $G_{1}(x, y, z), G_{2}(x, y, z) \in \mathbb{C}[t][x, y, z]$, respectively. If there exist $K, W, L \in \mathbb{C}[t][x, y, z]$ such that $K G_{1}+W G_{2}=z L$, and

(1) $G_{1}(x, y, 0) G_{2}(x, y, 0) \neq 0$.

(2) $\operatorname{gcd}\left(G_{1}(x, y, 0), G_{2}(x, y, 0)\right)=1$,

then either $z$ divides $K$ and $W$ or there exist $U_{1}, U_{2}, U_{3} \in \mathbb{C}[t][x, y, z]$ such that

$$
\mathrm{L}=U_{1} G_{1}(x, y, 0)+U_{2} G_{2}(x, y, 0)+z U_{3} .
$$


Proof. If $z$ divides $K$, then $z$ divides $W G_{2}$, and by (2) $z$ divides $W$. So let us assume that $z$ does not divides $K$, and let us denote by $G_{i}^{0}$ the polynomial $G_{i}(x, y, 0)$; similarly with $K^{0}, W^{0}$. Then, $K^{0} G_{1}^{0}+W^{0} G_{2}^{0}=0$. Since $G_{i}^{0} \neq 0$ and gcd $\left(G_{1}^{0}, G_{2}^{0}\right)=1$, then $G_{1}^{0}$ divides $W^{0}$ and $G_{2}^{0}$ divides $K^{0}$. Let $K^{0}=\Delta_{1} G_{2}^{0}, W^{0}=\Delta_{2} G_{1}^{0}$. So $\left(\Delta_{1}+\Delta_{2}\right) G_{1}^{0} G_{2}^{0}=0$, and since $G_{j}^{0} \neq 0$, one gets $\Delta_{1}+\Delta_{2}=0$. Now, we write

$$
K=K^{0}+z \bar{K}, \quad W=W^{0}+z \bar{W}, \quad G_{j}=G_{i}^{0}+z \bar{G}_{i},
$$

where $\bar{K}, \bar{W}, \bar{G}_{i} \in \mathbb{C}[t][x, y, z]$. Then, $K W_{1}+W G_{2}=z\left(G_{1}^{0} \bar{K}+G_{2}^{0} \bar{W}+z\left(\bar{K} \bar{G}_{1}+\bar{W} \bar{G}_{2}\right)\right)$.

Theorem 4.5. The rational curve $\overline{\mathcal{C}}$, output by the algorithn, and $\mathcal{C}$ have the same points at infinity, and $\operatorname{deg}(\bar{C})=\operatorname{deg}(\mathcal{C})$.

Proof. The fact on the degree follows from Lemmas 4.3 and 4.4, once the structured at infinity will be proved. For the rest of the proof, let $H^{*}(t, x, y, z), F(x, y, z), S_{1}(x, t), S_{2}(y, t), A_{1}(x), A_{2}(y), B_{1}(x, t):=q_{1}(t) x-p_{1}(t), B_{2}(y, t):=q_{2}(t) y-p_{2}(t)$ as in the algorithm, and let $R_{i}$ be the remainder of the division of $S_{i}$ by $A_{i}$. By Lemma $4.2, q_{1}(t)=\lambda q_{2}(t)$, with $\lambda \in \mathbb{L}^{*}$. By Lemma 4.3, $\operatorname{deg}_{i}\left(B_{1}\right)=\operatorname{deg}_{\mathrm{t}}\left(B_{2}\right)=\operatorname{deg}(F)=d$ and, by Step 10 of the algorithm, we can assume w.l.o.g. that gcd $\left(q_{1}, p_{1}\right)=$ $\operatorname{gcd}\left(q_{2}, p_{2}\right)=1$. So,

$$
\overline{\mathcal{P}}^{H}(t):=\left(\lambda^{-1} p_{1}(t): p_{2}(t): q_{2}(t)\right)
$$

parametrizes the projective closure of $\overline{\mathcal{C}}$. Furthermore, since $\operatorname{deg}\left(\boldsymbol{p}_{i}\right) \leqslant \operatorname{deg}\left(q_{2}\right)$, then all points of $\overline{\mathcal{C}}$ at infinity are reachable by $\overline{\mathcal{P}}^{H}(t)$ (see Sendra, 2002). In addition, we note that

$$
\operatorname{deg}_{\{x, y, z\}}\left(H^{*}\right)=d-2, \quad \operatorname{deg}\left(A_{j}\right)=d(d-2)-1, \quad \operatorname{deg}_{\{x, y\}}\left(R_{j}\right) \leqslant d(d-2)-2 .
$$

Moreover, if $m^{H}(x, y, z, w)$ denotes the homogenization of $m(x, y, w)$ as a polynomial in $c[w][x, y]$, we have that

$$
\begin{aligned}
& \bar{S}_{1}^{H}(x, z, t)=\operatorname{Res}_{y}\left(H^{*}(t, x, y, z), F(x, y, z)\right)=B_{1}^{H}(x, z, t) A_{1}^{H}(x, z)+R_{1}^{H}(x, z, t) z^{n_{1}}, \\
& \bar{S}_{2}^{H}(y, z, t)=\operatorname{Res}_{x}\left(H^{*}(t, x, y, z), F(x, y, z)\right)=B_{2}^{H}(y, z, t) A_{2}^{H}(y, z)+R_{2}^{H}(y, z, t) z^{n_{2}},
\end{aligned}
$$

where $n_{j}+\operatorname{deg}\left(R_{j}^{H}\right)=d(d-2), j=1,2$. So $n_{j} \geqslant 2$. Also, we denote by $\bar{C}_{\infty}$ and $\mathcal{C}_{\infty}$ the set of points at infinity $\bar{C}$ and $\mathcal{C}$ respectively. By resultant properties, there exist polynomials $M_{1}, N_{1}, M_{2}, N_{2} \in[t, x, y, z]$ such that

$$
M_{i} H^{*}+N_{i} F=\bar{S}_{j}^{H}, i=1,2 .
$$

So,

$$
y A_{2}^{H} S_{1}^{H}-\lambda x A_{1}^{H} S_{2}^{H}=z A_{1}^{H} A_{2}^{H}\left(\lambda x p_{2}-y p_{1}\right)+z^{n_{3}} R_{3} .
$$

where $n_{3} \geqslant 2$ and $R_{3}$ a polynomial; namely $z^{\Omega_{3}} R_{3}=y A_{2} z^{\Omega_{1}} R_{1}^{H}-\lambda x A_{1} z^{n_{2}} R_{2}^{H}$. On the other hand, if $K=y A_{2}^{H} M_{1}-\lambda x A_{1}^{H} M_{2}$ and, $W=y A_{2}^{H} N_{1}-\lambda x A_{1}^{H} N_{2}$, then

$$
y A_{2}^{H} S_{1}^{H}-\lambda x A_{1}^{H} S_{2}^{H}=K(x, y, z, t)\left(H_{1}+t H_{2}\right)+W(x, y, z, t) F .
$$

Therefore, $z$ divides the right hand side of the above equation. We now check that $H_{1}+t H_{2}$ and $F$ satisfy the hypothesis of Lemma 4.4. Since $F$ is irreducible and non-linear, $F(x, y, 0) \neq 0$. Moreover, if $H_{1}(x, y, 0)+t H_{2}(x, y, 0)=0$ then $H_{2}(x, y, 0)=0$ and this implies that $\mathcal{D}_{2}$ contains all the points at infinity of $\mathcal{C}^{h}$, which is a contradiction. Finally, if $\operatorname{gcd}\left(H_{1}(x, y, 0)+t H_{2}(x, y, 0), F(x, y, 0)\right) \neq 1$, then $\operatorname{gcd}\left(H_{2}(x, y, 0), F(x, y, 0)\right) \neq 1$, and this implies that $\mathcal{D}_{2}$ and $c^{h}$ share points at infinity. Therefore, applying Lemma 4.4 , one deduces that either there exist polynomials $M_{3}, N_{3} \in \mathbb{C}[t][x, y, z]$ such that

$$
M_{3} H^{*}+N_{3} F=A_{1}^{H} A_{2}^{H}\left(\lambda x p_{2}-y p_{1}\right)+z^{n_{4}} R_{3},
$$

where $n_{4} \geqslant 1$, or there exist polynomials $U_{1}, U_{2}, U_{3} \in \mathbb{C}[t][x, y, z]$ such that

$$
U_{1} H^{*}(\mathrm{t}, x, y, 0)+U_{2} F(x, y, 0)+z U_{3}=A_{1}^{H} A_{2}^{H}\left(\lambda x p_{2}-y p_{1}\right)+z^{n_{4}} R_{3} \text {. }
$$

In this situation, using $\bar{C}_{\infty} \subset \overline{\mathcal{P}}^{H}\left(C^{7}\right)$, we first observe that Card $\left(\bar{C}_{\infty}\right)$ is less or equal to the number of different roots of $q_{2}$ (t) and, by Lemma 4.2 , this number is less or equal to $\operatorname{Card}\left(\mathcal{C}_{\infty}\right)$. So, $\operatorname{Card}\left(\overline{\mathcal{C}}_{\infty}\right) \leqslant$ Card $\left(\mathcal{C}_{\infty}\right)$. Now, we prove that $\mathcal{C}_{\infty}, \overline{\mathcal{C}_{\infty}}$, from where one concludes the proof. Let $P=\left(x_{0}: y_{0}: 0\right) \in \mathcal{C}_{\infty}$, and let $t_{0}$ be the root of $q_{2}$ generated by $P$ (see Lemma 4.2). So, $H^{*}\left(t_{0}, x_{0}, y_{0}, 0\right)=F\left(x_{0}, y_{0}, 0\right)=0$. Applying the corresponding equality above, and using that $n_{4} \geqslant 1$, we get

$$
A_{1}^{H}\left(x_{0}, 0\right) A_{2}^{H}\left(y_{0}, 0\right)\left(\lambda x_{0} p_{2}\left(t_{0}\right)-y_{0} p_{1}\left(t_{0}\right)\right)=0 \text {. }
$$

Moreover, since $\{1: 0: 0),\{0: 1: 0) \notin C^{h}$ then $x_{0} y_{0} \neq 0$, and hence $A_{1}^{H}\left(x_{0}, 0\right) A_{2}^{H}\left(y_{0}, 0\right) \neq 0$. So, $\lambda_{0} x_{0} p_{2}\left(t_{0}\right)=y_{0} p p_{1}\left(t_{0}\right)$. ln addition, $p_{1}\left(t_{0}\right) p_{2}\left(t_{0}\right) \neq 0$ because $\operatorname{gcd}\left(q_{2}, p_{1}\right)=1=\operatorname{gcd}\left(q_{2}, p_{2}\right)$. Therefore, 


$$
\begin{aligned}
\overline{\mathcal{P}}^{H}\left(t_{0}\right) & =\left(\lambda^{-1} p_{1}\left(t_{0}\right): p_{2}\left(t_{0}\right): 0\right) \\
& =\left(y_{0} \lambda^{-1} p_{1}\left(t_{0}\right): y_{0} p_{2}\left(t_{0}\right): 0\right)=\left(x_{0} p_{2}\left(t_{0}\right): y_{0} p_{2}\left(t_{0}\right): 0\right)=\left(x_{0}: y_{0}: 0\right)=\mathrm{P} .
\end{aligned}
$$

Corollary 4.6. The asymptotes of $\mathcal{C}$ and $\bar{C}$ are parallel.

Proof. It follows from Theorem 3, p. 42, in Coolidge (1959).

\section{Displaying examples}

In this section we present several examples to illustrate the algorithm. We note that the algorithm did no require perturbing $H^{*}$ in any of the examples.

These examples have been computed in Maple. Let us give some details on how the computations have been done. As mentioned in Remark 4.1(1), we work symbolically with the exceptions of Steps 1 and 4 . So the input polynomial $f(x, y)$, and the tolerance $\epsilon$, are converted (if necessary) into a rational representation, and we only pass to floating point arithmetic when computing the $\epsilon$-singularities and the $\epsilon$-simple points. For the $\epsilon$-singularities computation we proceed as follows (similarly for the $\epsilon$-simple points): $f$ is converted into a floating-point coefficient polynomial; then, for each $k \in\{2, \ldots, \operatorname{deg}(\mathcal{C})\}$ we choose two partial derivatives of $f$ of order $k-1$ and we apply Maple commands to solve the bivariate system defined by them; more precisely we consider the sequence of Maple commands evalf(allvalues(solve $\left.\left(\mathrm{pol}_{1}(x, y), \mathrm{pol}_{2}(x, y)\right)\right)$ ); once this set of solutions has been computed, we find the subset of solutions satisfying that the modulo of all partial derivatives, of order less or equal to $k-1$, evaluated at the solution, is smaller or equal to $\epsilon\|f\|$; we consider next the union of all the resulting sets and we perform the cluster distribution; finally we transform the cluster representatives to rational representation.

We write here, and in the next section, $\bar{f}$ and $\overline{\mathcal{P}}_{(t)}$ with 10-digits floating point coefficients, but the executions have been performed with the exact version; the precise data can be seen at www $2 . u a h . e s / \operatorname{sperez} / \sec 5 \sec 6$.pdf.

Example 5.1. Let $\epsilon=\frac{1}{100}$ and $\mathcal{C}$ the curve of proper degree 5 defined by (see Fig. 3):

$$
\begin{aligned}
f(x, y)= & -x^{2} y+\frac{17465}{117409} x y^{3}+\frac{1741}{281822} y^{5}-\frac{12539}{19281} x y+\frac{4167}{639011} x^{3} y-\frac{24571}{35273} x y^{2}+\frac{38146}{217805} x^{2} y^{2} \\
& -\frac{15409}{48541} x^{3}+\frac{5933}{390846} x y^{4}+\frac{4465}{126254} y^{4}-\frac{61558}{172857} x^{2}-\frac{6938}{29505} y^{2}+\frac{4167}{639011} x^{3} y^{2}+\frac{4167}{639011} x^{4} \\
& +\frac{4167}{639011} x^{4} y+\frac{3021}{339304} x^{2} y^{3}+\frac{4167}{639011} x^{5}-\frac{11559}{74969} y^{3}+\frac{182}{2763067}
\end{aligned}
$$

First we compute the $\epsilon$-singularities of $\mathcal{C}$. $\operatorname{Sing}_{\epsilon}(\mathcal{C})=\mathcal{S}_{1} \cup \mathcal{S}_{2} \cup \mathcal{S}_{3}$ where:

$$
\begin{aligned}
\mathcal{S}_{1}=\left\{Q_{1}\right. & =(-3.999854219,2.000094837), Q_{2}=(0 ., 0 .), \\
Q_{3} & =(0.9998153818,-2.999388343), \\
Q_{4} & =(-2.001190360+0.05414244305 i, 3.001898191-0.08039416354 i), \\
Q_{5} & =(-1.980207988,3.002780607), Q_{6}=(-2.019931003,2.997118979), \\
Q_{7} & =(-2.001190360-0.05414244305 i, 3.001898191+0.08039416354 i)\}, \\
\mathcal{S}_{2}=\left\{Q_{8}\right. & =(-2.000000001,3.000000001)\} . \\
\mathcal{S}_{3}=\emptyset &
\end{aligned}
$$

Moreover, the cluster decomposition of the singular locus is (see Fig. 2, Left):

$$
\begin{aligned}
& \text { Cltuster }_{2}\left(Q_{1}\right)=\left\{Q_{1}\right\}, \quad \text { Cluster }_{2}\left(Q_{2}\right)=\left\{Q_{2}\right\}, \quad \text { Cluster }_{2}\left(Q_{3}\right)=\left\{Q_{3}\right\} \text { and } \\
& \text { eltister }_{3}\left(Q_{8}\right)=\left\{Q_{4}, Q_{5}, Q_{6}, Q_{7}, Q_{8}\right\} .
\end{aligned}
$$

We observe that $\mathcal{C}$ is $\epsilon$-rational. Following Step 4 in the algorithm we obtain two $\epsilon$-ramification points, namely $P_{1}=$ $(3.437938023,4.260660564), P_{2}=(7.712891931,1.573609575)$. We note that these points are not in the cluster of each other and they are not in the clusters of the cluster decomposition of the singular locus (see Fig. 2, Right).

Finally, the algorithm outputs the parametrization $\overline{\mathcal{P}}(t)=\left\langle\left(\frac{\bar{p}_{1}(t)}{\bar{q}(t)}, \overline{\bar{p}_{2}(t)}\right)\right.$ where (see Fig. 3 to compare the input and the output curves): 

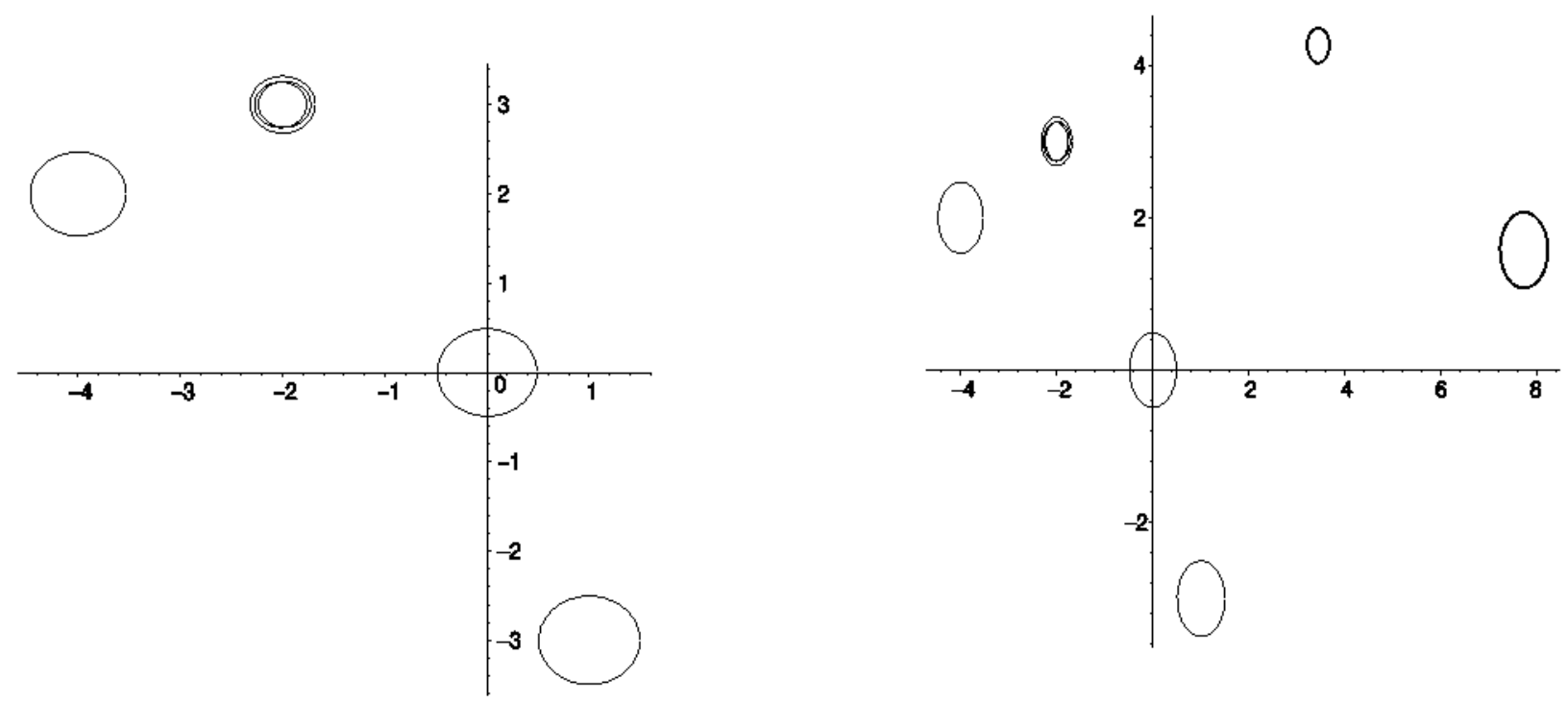

Fig. 2. Left: Cluster decomposition of the singular locus. Right: Cluster decomposition of the singular locus with two $\epsilon$-ranification points.
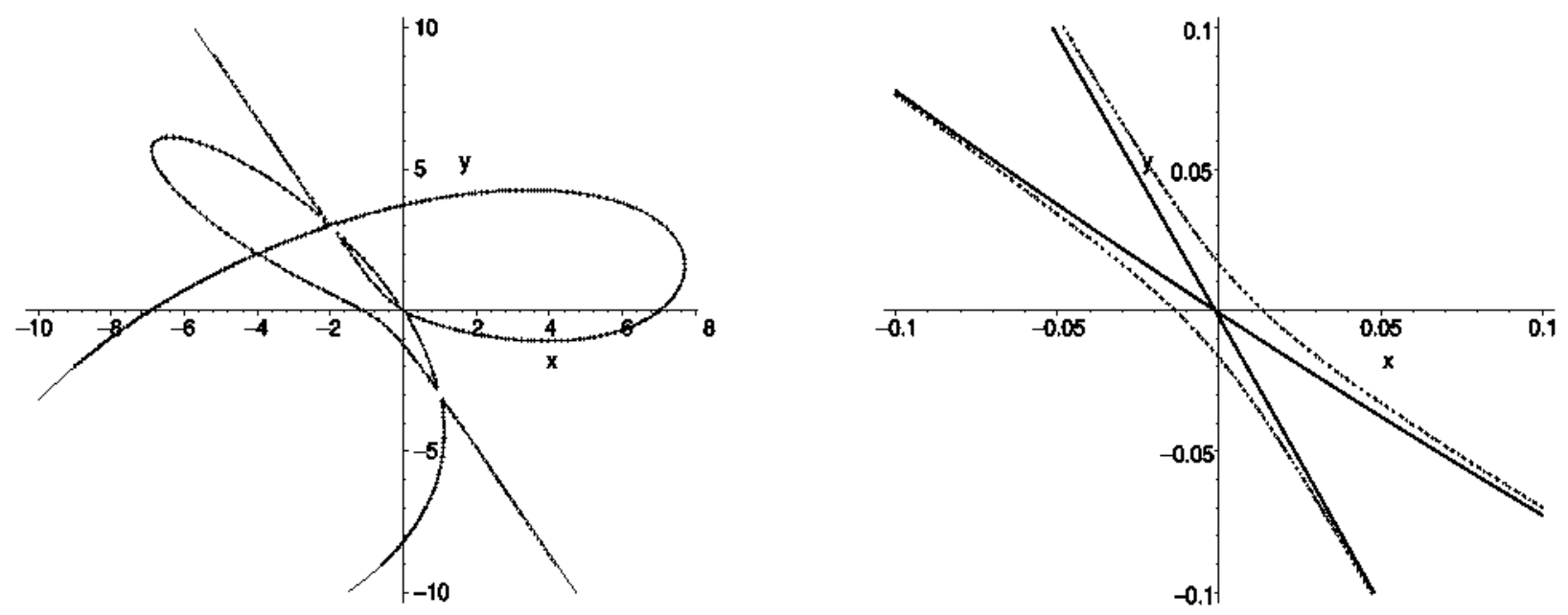

Fig. 3. Left: Input (in dots) and output curve in Example 5.1. Right: A zoom at the origin.

$$
\begin{aligned}
\bar{p}_{1}(t)= & 0.1928498375 \cdot 10^{551} t^{2}+0.1974296234 \cdot 10^{549} t^{4}-0.3199304792 \cdot 10^{547} t^{5} \\
& +0.4048447557 \cdot 10^{550} t+0.1193911126 \cdot 10^{551} t^{3}-0.8374467974 \cdot 10^{549}, \\
\bar{p}_{2}(t)= & 0.2075974869 \cdot 10^{550}+0.8229900424 \cdot 10^{548} t^{4}-0.1401409004 \cdot 10^{551} t \\
& -0.4936881030 \cdot 10^{550} t^{3}-0.1914613475 \cdot 10^{551} t^{2}+0.5662842458 \cdot 10^{547} t^{5}, \\
\bar{q}(t)= & -0.1205298833 \cdot 10^{550}-0.1863676648 \cdot 10^{550} t-0.1381087143 \cdot 10^{550} t^{2} \\
& -0.5167655604 \cdot 10^{549} t^{4}-0.1020066715 \cdot 10^{550} t^{3}+0.5236942518 \cdot 10^{546} t^{5} .
\end{aligned}
$$

Example 5.2. Let $\epsilon=\frac{4}{1000}$ and $\mathcal{C}$ the curve of proper degree 6 defined by (see Fig. 5):

$$
\begin{aligned}
f(x, y)= & \frac{3013}{3735} x y-\frac{11}{20} x+y-\frac{31}{75} x y^{3}+\frac{1}{150} x^{4} y^{2}+\frac{1}{25} x^{3} y^{3}+\frac{41}{75} x^{2} y^{2}+\frac{17}{150} x^{3} y^{2}+\frac{1}{20} x y^{4}+\frac{1}{150} x^{5} y \\
& -\frac{7}{100} x^{2} y^{4}-\frac{1}{30} x^{2} y-\frac{8}{15} y^{3}+\frac{1}{150} x^{6}+\frac{1}{150} y^{6}-\frac{67}{100} x^{2}-\frac{53}{300} x^{4}+\frac{9}{25} y^{4}-\frac{47}{100} x^{3} \\
& -\frac{1}{150} x^{5}+\frac{1}{150} x^{2} y^{3}+\frac{19}{50} x y^{2}+\frac{1}{150} x^{4} y-\frac{1}{50} x y^{5}-\frac{8}{75} x^{3} y-\frac{91}{150} y^{2}-\frac{1}{25} y^{5}+\frac{1}{37350}
\end{aligned}
$$




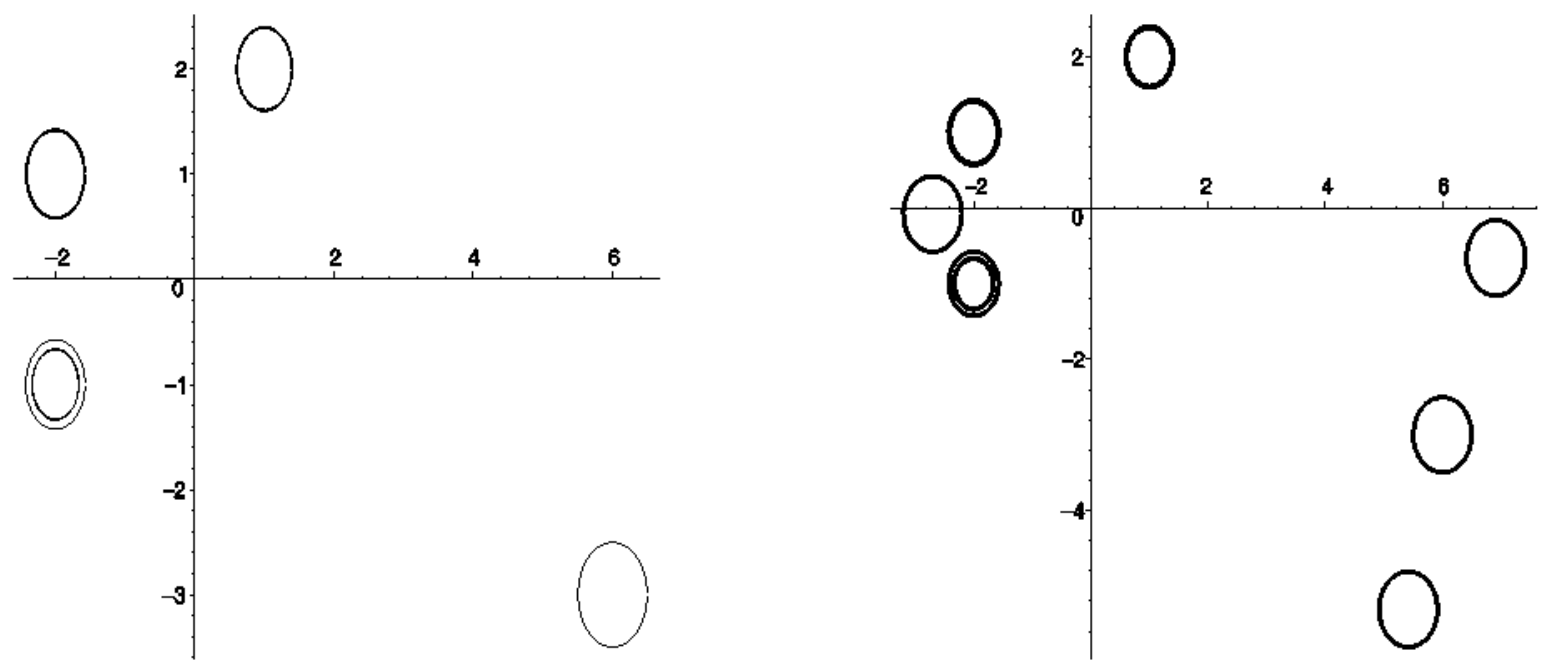

Fig. 4. Left: Cluster decomposition of the singular locus, Right: Cluster decomposition of the singular locus with two $\epsilon$-ramification points.

We get the $\epsilon$-singular locus $\operatorname{Sing}_{\epsilon}(\mathcal{C})=\mathcal{S}_{1} \cup \mathcal{S}_{2} \cup \mathcal{S}_{3}$ where

$$
\begin{aligned}
\mathcal{S}_{1}=\left\{Q_{1}\right. & =(-1.994232333,1.005043048), \\
Q_{2} & =(-2.000005299+0.005645280797 i,-1.000026945-0.0002822677587 i), \\
Q_{3} & =(-2.000014217+0.004619269427 i, 1.000004775-0.003559494332 i), \\
Q_{4} & =(-2.003547061,-1.006293429), Q_{5}=(-2.005740475,0.9948974977), \\
Q_{6} & =(-1.996418580,-0.9936748962), \\
Q_{7} & =(-2.000014217-0.004619269427 i, 1.000004775+0.003559494332 i), \\
Q_{8} & =(-2.000005299-0.005645280797 i,-1.000026945+0.002822677587 i), \\
Q_{9} & =(1.000036272+0.008596901071 i, 2.000017052-0.003059926359 i), \\
Q_{10} & =(5.999999669,-2.999998564), \\
Q_{11} & =(1.000036272-0.008596901071 i, 2.000017052-0.003059926359 i), \\
Q_{12} & \left.=(0.9978910941,1.994329680), Q_{13}=(1.002094534,2.005650021)\right\}, \\
S_{2}=\left\{Q_{14}\right. & \left.=(-2.000000001,1 .), Q_{15}=(-2 .,-1.000000005), Q_{16}=(1 ., 2 .)\right\} \\
S_{3}= &
\end{aligned}
$$

The singular cluster decomposition is (see Fig. 4, Left):

$$
\begin{aligned}
& \text { Cluster } 2\left(Q_{10}\right)=\left\{Q_{10}\right\}, \quad \text { Cluster }\left(Q_{14}\right)=\left\{Q_{1}, Q_{3}, Q_{5}, Q_{7}, Q_{14}\right\} \\
& \text { Cluster },\left(Q_{15}\right)=\left\{Q_{2}, Q_{4}, Q_{6}, Q_{8}, Q_{15}\right\}, \text { and } \text { Cluster }_{3}\left(Q_{16}\right)=\left\{Q_{9}, Q_{11}, Q_{12}, Q_{13}, Q_{16}\right\} .
\end{aligned}
$$

We observe that $\mathcal{C}$ is $\epsilon$-rational. In Step 4 we obtain three $\epsilon$-ramification points: $P_{1}=(-1.330235522,0.9268173641)$. $P_{2}=(-1.979908167,0.02661222172)$, and $P_{3}=(-2.700785807,-0.07757312293)$. We note that these points are not in the cluster of each other and they are not in the clusters of the $\epsilon$-singularities (see Fig. 4, Right).

The algorithm outputs the parametrization $\overline{\mathcal{P}}(t)=\left(\frac{\bar{p}_{1}(t)}{\bar{q}(t)}, \frac{\bar{p}_{2}(t)}{\bar{q}(t)}\right)$ where (see Fig. 5 to compare the input and the output curves):

$$
\begin{aligned}
\bar{p}_{1}(t)= & -0.4665969363 \cdot 10^{910} t^{6}+0.1734681470 \cdot 10^{912} t^{5}+0.8664051685 \cdot 10^{914} t \\
& -0.4159434177 \cdot 10^{914} t^{3}-0.4505650348 \cdot 10^{915}+0.2152577377 \cdot 10^{915} t^{2}+0.7566892493 \cdot 10^{912} t^{4}, \\
\bar{p}_{2}(t)= & 0.2378210112 \cdot 10^{914} t^{3}+0.2759395174 \cdot 10^{914} t+0.2196326945 \cdot 10^{912} t^{5}+0.4655361031 \cdot 10^{914} t^{2} \\
& -0.4970648521 \cdot 10^{913} t^{4}-0.1850888359 \cdot 10^{915}-0.2330404339 \cdot 10^{910} t^{6}, \\
\bar{q}(t)= & -0.2692822852 \cdot 10^{914} t^{3}+0.3583267610 \cdot 10^{913} t^{4}-0.1530968486 \cdot 10^{912} t^{5} \\
& +0.2330431163 \cdot 10^{910} t^{6}+0.6095885650 \cdot 10^{915}+0.5717788848 \cdot 10^{915} t-0.2358118980 \cdot 10^{914} t^{2} .
\end{aligned}
$$



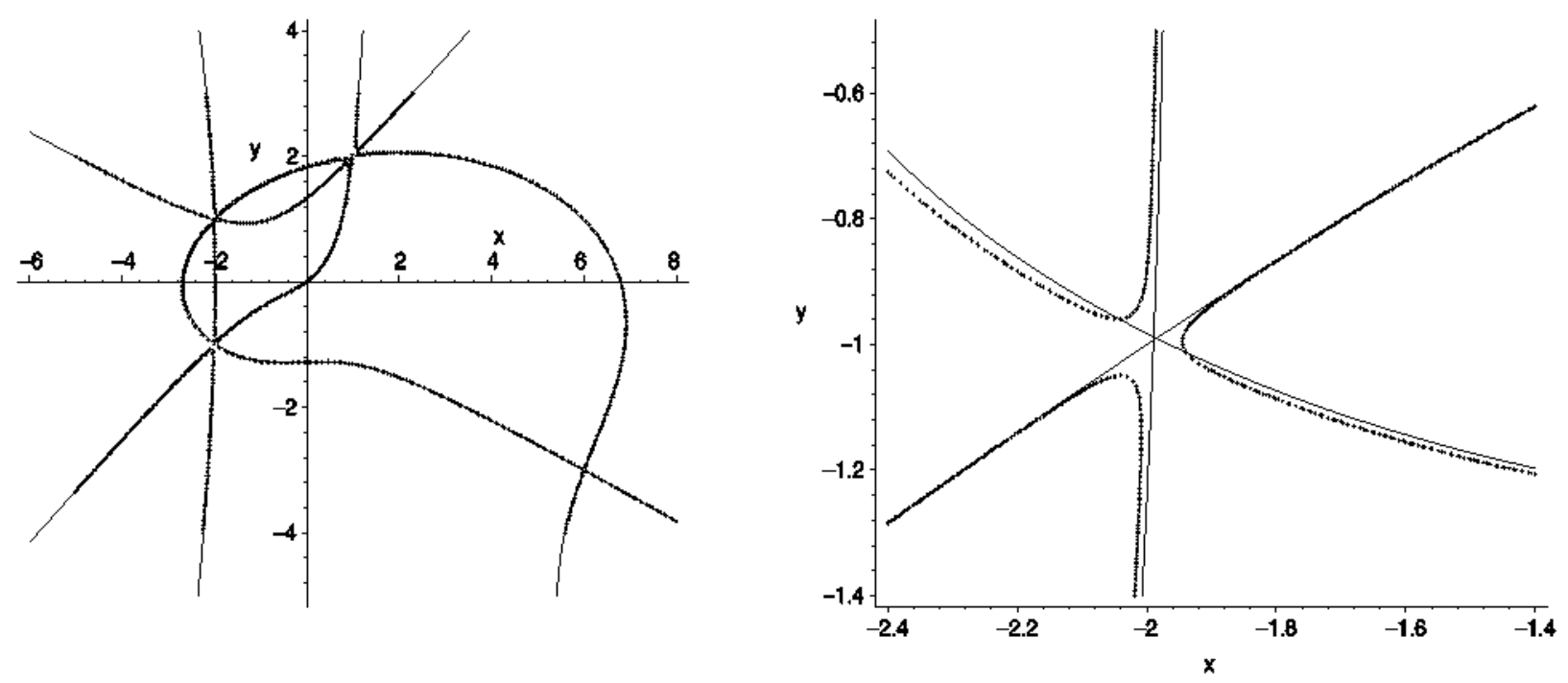

Fig. 5. Lelt: Input (in dots) and output curve in Example 5.2. Fighl: A zoom at $(-2,-1)$,

Example 5.3. Let us consider a surface with implicit equation

$$
\begin{aligned}
F(x, y, z)= & \frac{1}{100} x+\frac{171201}{100} y+432 x^{2} z y-216 x y^{2} z+\frac{1}{100} z-\frac{17119999}{10000} z^{2}+\frac{1}{10000} x z+\frac{1920001}{10000} y z \\
& +\frac{4320001}{10000} y^{2}-\frac{7999999}{1000000} y^{2} z-\frac{1439999}{10000} x y+\frac{856000001}{1000000} x^{2} z+\frac{1}{10000} x^{2}+\frac{144000001}{1000000} z^{2} x \\
& +864 z^{4}-192 z^{3}+1296 z^{6}-1728 z^{5}-1284 x^{3}-2576 x z y+144 z^{3} x y-96 z^{2} x y-3888 z^{4} x \\
& +3936 z^{2} x^{2}-72 z^{3} x^{2}-72 z^{3} y^{2}+2592 z^{3} y+48 z^{2} y^{2}-1296 z^{2} y+864 x z^{3}-216 x^{3} z \\
& +\frac{12000001}{1000000} x^{2} y-\frac{59999999}{1000000} x y^{2}-4 x^{3} y+6 x^{2} y^{2}-4 x y^{3}+\frac{36000001}{1000000} y^{3}+x^{4}+y^{4}
\end{aligned}
$$

We consider the planar sectioning problem. The intersection of the surface with planes $z=1 / 2^{i}, i=1, \ldots, 10$ generates a family of 10 curves of proper degree 4 that are not rational. However, our algorithm shows that 9 of them are $\epsilon$-rational. In what follows, we present details of the application of the approximate parametrization algorithm to one of the curves of this family. Namely the curve defined by the polynomial (see Fig. 6):

$$
\begin{aligned}
f(x, y)= & -\frac{395959799}{4000000} x+\frac{36160201}{20000} y+\frac{862000201}{2000000} y^{2}-\frac{14379999}{10000} x y+\frac{2806000201}{2000000} x^{2}-1392 x^{3} \\
& +\frac{228000001}{1000000} x^{2} y-\frac{167999999}{1000000} x y^{2}-4 x^{3} y+6 x^{2} y^{2}-4 x y^{3}+\frac{36000001}{1000000} y^{3}+x^{4}+y^{4}-\frac{17269799}{40000} .
\end{aligned}
$$

Let $\epsilon=\frac{1}{100}$. Sing $\epsilon(\mathcal{C})=\mathcal{S}_{1} \cup \mathcal{S}_{2} \cup \mathcal{S}_{3}$ where

$$
\begin{aligned}
& \mathcal{S}_{1}=\left\{Q_{1}=(0.9433279517,-6.369071364)\right\}, \\
& \mathcal{S}_{2}=\left\{Q_{2}=(-0.6522149822-0.9122043803 i,-14.99598555+3.758226746 i)\right\}, \\
& \mathcal{S}_{3}=\left\{Q_{3}=(-0.6522149822+0.9122043803 i,-14.99598555-3.758226746 i)\right\} .
\end{aligned}
$$

Moreover, the cluster decomposition of the singular locus is:

$$
\text { cltister }_{2}\left(Q_{1}\right)=\left\{Q_{1}\right\}, \quad \text { Cluster }_{2}\left(Q_{2}\right)=\left\{Q_{2}\right\} . \quad \text { Eltister }_{2}\left(Q_{3}\right)=\left\{Q_{3}\right\} \text {. }
$$

We observe that $C$ is $\epsilon$-rational. Following Step 4 in the algorithm we obtain the $\epsilon$-ramification point, namely $P_{1}=$ $\{420.8571421,-157.1835301\}$. We note that this point is not in the clusters of the $\epsilon$-singularities.

Finally, the algorithm outputs the parametrization $\overline{\mathcal{P}}(t)=\left(\frac{\bar{p}_{1}(t)}{\bar{q}(t)}, \frac{\bar{p}_{2}(t)}{\bar{q}(t)}\right)$ where (see Fig. 6):

$$
\begin{aligned}
\bar{p}_{1}(t)= & 0.3678784753 \cdot 10^{346} t^{4}+0.1244267377 \cdot 10^{350} t^{2}+0.1165894081 \cdot 10^{352} \\
& -0.3494866166 \cdot 10^{348} t^{3}-0.1967543000 \cdot 10^{351} t
\end{aligned}
$$




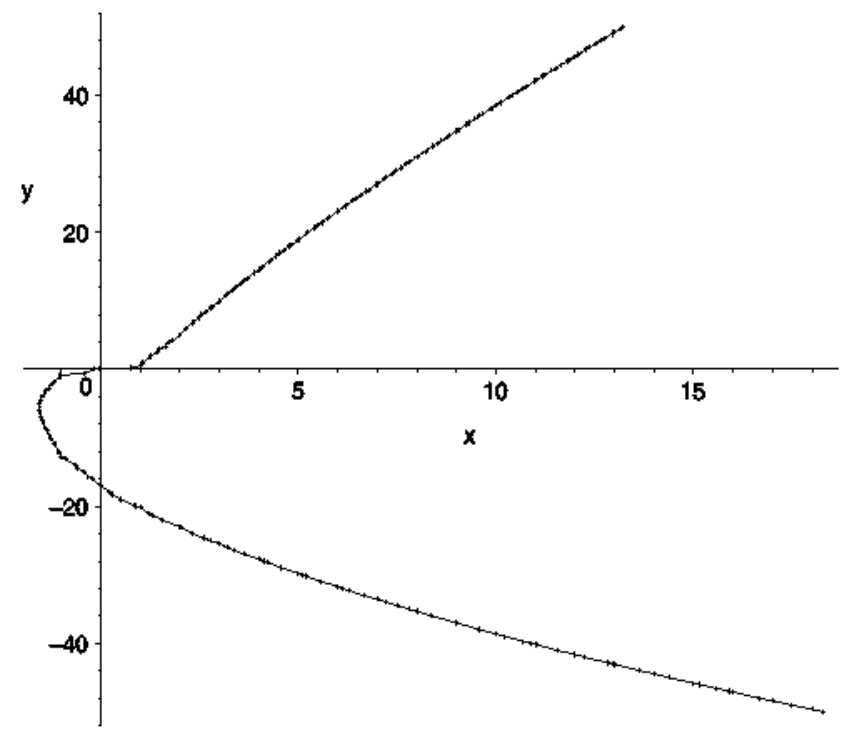

Fig. 6. Input (in dots) and output curve in Example 5.3.

$$
\begin{aligned}
\bar{p}_{2}(t)= & 0.6170290181 \cdot 10^{349} t^{2}-0.1699115220 \cdot 10^{348} t^{3}+0.6038177598 \cdot 10^{351} \\
& +0.1755842639 \cdot 10^{346} t^{4}-0.9964982725 \cdot 10^{350} t \\
\bar{q}(t)= & 0.7090236056 \cdot 10^{346} t^{4}+0.2453189759 \cdot 10^{352}+0.2502344903 \cdot 10^{350} t^{2} \\
& -0.6878413782 \cdot 10^{348} t^{3}-0.4045978049 \cdot 10^{351} t
\end{aligned}
$$

\section{Empirical analysis of the error}

We start describing briefly the theoretical setting. For that purpose, we will follow Aliprantis and Border (2006); in particular its Section 3.14. Let $(X, d)$ be a metric space. For $\emptyset \neq B \subset X$ and $a \in X$ we define

$$
d(a, B)=\inf _{b=B}\{d(a, b)\} .
$$

Moreover, for $A, B \subset X \backslash\{\emptyset\}$ we define

$$
H_{d}(A, B)=\max \left\{\sup _{a \in A}\{d(a, B)\}, \sup _{b \in B}\{d(b, A)\}\right\} .
$$

By convection $H_{d}(\emptyset, \emptyset)=0$ and, for $\emptyset \neq A \subset X, H_{d}(A, \emptyset)=\infty$. The function $H_{d}$ is called the Hausdorff distance induced by $d$. The Hausdorff distance defines a metric on the close subsets of $X$ (see lemma 3.57 in Aliprantis and Border, 2006).

Now, we consider the metric spaces $\left(\mathbb{R}^{2}, d_{e}\right) \subset\left(\mathbb{C}^{2}, d_{u}\right)$ where $d_{e}$ and $d_{u}$ are the usual Euclidean and unitary metrics, respectively. Since $\left.d_{u}\right|_{\mathbb{R}^{2}}=d_{e}$, in the sequel we denote by $d$ both distances and by $H$ the Hausdorff distance associated to $d$. Moreover, for $A \subset \mathbb{C}^{2}$ we denote by $A^{\mathbb{E}}$ the set $A \cap \mathbb{E}^{2}$. Then, for $A, B \subset \mathbb{C}^{2}$ we consider $H\left(A^{\mathbb{R}}, B^{\mathbb{E}}\right)$; one might also consider $H(A, B), H\left(A^{\mathbb{E}}, B\right), H\left(A, B^{\mathbb{R}}\right)$.

In this situation, let $\mathcal{C}$ and $\bar{C}$ be the input and output curves of our algorithm. In addition, let $f(x, y)$ and $\bar{f}(x, y)$ be the defining polynomials of $\mathcal{C}$ and $\overline{\mathcal{C}}$, respectively, and let $\overline{\mathcal{P}}(t)=\left(\bar{p}_{1}(t), \bar{p}_{2}(t)\right) \in \mathbb{R}(t)^{2}$ be the parametrization of $\overline{\mathcal{C}}$ output by our algorithm. In general the Hausdorff distance can be infinity, but in our case we can ensure that it is finite.

Lemma 6.1. $H\left(\mathcal{C}^{\mathbb{E}}, \overline{\mathcal{C}}^{\mathbb{E}}\right)<\infty$.

Proof. By Theorem 4.5 and Corollary $4.6, \mathcal{C}^{\mathbb{R}}, \overline{\mathcal{C}}^{\mathbb{R}}$ are either bounded or all their points at infinity define asymptotes that are parallel. If the first case, the lemma follows from lemma 3.58 in Aliprantis and Border (2006). Otherwise, let $\mathcal{L}_{1} \ldots, \mathcal{L}_{n}$ be the real asymptotes of $\mathcal{C}$ and let $\overline{\mathcal{L}}_{1}, \ldots, \overline{\mathcal{L}}_{n}$ be the real asymptotes of $\overline{\mathcal{C}}$, with $\mathcal{L}_{i} \| \overline{\mathcal{L}}_{i}$. Let $\rho>0$, then there exists a compact ball $B$, such that for every $P \in C^{\mathbb{P}} \cap\left(\mathbb{R}^{2} \backslash B\right), d\left(P, \bar{C}^{\mathbb{P}}\right)<\rho$. Then,

$$
H\left(C^{\mathbb{E}}, \bar{C}^{\mathbb{E}}\right) \leqslant \max \left\{H\left(\mathcal{C}^{\mathbb{E}} \cap B, \overline{\mathcal{C}}^{\mathbb{R}} \cap B\right), 2 \rho+H\left(\mathcal{L}_{1}, \overline{\mathcal{L}}_{1}\right), \ldots, 2 \rho+H\left(\mathcal{L}_{n}, \overline{\mathcal{L}}_{n}\right)\right\}
$$

that is finite because of Lemma 3.58 by Aliprantis and Border (2006). 
In order to study $H\left(\mathcal{C}^{\mathbb{P}}, \overline{\mathcal{C}}^{\mathbb{E}}\right)$, we consider the normal line to $\overline{\mathcal{C}}$ at the generic point $\overline{\mathcal{P}}(\boldsymbol{t})$ :

$$
\mathcal{L}_{1}(t, s)=\left(\bar{p}_{1}(t)+s \frac{-\bar{p}_{2}^{\prime}(t)}{\sqrt{\bar{p}_{1}^{\prime}(t)^{2}+\bar{p}_{2}^{\prime}(t)^{2}}}, \bar{p}_{2}(t)+s \frac{\bar{p}_{1}^{\prime}(t)}{\sqrt{\bar{p}_{1}^{\prime}(t)^{2}+\bar{p}_{2}^{\prime}(t)^{2}}}\right) .
$$

as well as the normal line to $\mathcal{C}$ at the generic point $(a, b) \in \mathcal{C}^{\mathbb{R}}$ :

$$
\mathcal{L}_{2}(a, b, s)=\left(a+s \frac{\mathrm{n}_{1}(a, b)}{N(a, b)}, b+s \frac{\mathrm{n}_{2}(a, b)}{\mathrm{N}(a, b)}\right),
$$

where $\left(n_{1}(a, b), n_{2}(a, b)\right)=\nabla f(a, b)$ and $N(a, b)=+\sqrt{n_{1}(a, b)^{2}+n_{2}(a, b)^{2}}$. Moreover, we introduce the polynomials

$$
\mathcal{D}_{1}(t, s)=f\left(\mathcal{L}_{1}(t, s)\right) \in \overline{\mathbb{R}(t)}[s], \quad \mathcal{D}_{2}(a, b, s)=\bar{f}\left(\mathcal{L}_{2}(a, b, s)\right) \in \mathbb{C}(\overline{\mathcal{C}})[s] .
$$

where $\overline{\mathbb{R}(t)}$ denotes the algebraic closure of $\mathbb{R}(t)$ and $\mathfrak{C}(\overline{\mathcal{C}})$ the field of rational functions over $\overline{\mathcal{C}}$. For every $\mathfrak{t}_{0} \in \mathbb{R}$, such that $\mathcal{D}_{1}\left(t_{0}, s\right)$ is well defined and has real roots,

$$
d\left(\overline{\mathcal{P}}\left(t_{0}\right), c^{\mathbb{R}}\right) \leqslant \rho_{1}^{\mathbb{R}_{1}}\left(t_{0}\right), \quad \text { where } \rho_{1}^{\mathbb{R}}\left(t_{0}\right)=\min \left\{\left|s_{0}\right| / \mathcal{D}_{1}\left(t_{0}, s_{0}\right)=0 \text { and } s_{0} \in \mathbb{R}\right\} .
$$

and for every $\left(a_{0}, b_{0}\right) \in \mathcal{C}^{\mathbb{R}}$, such that $\mathcal{D}_{2}\left(a_{0}, b_{0}, s\right)$ is well defined and has real roots,

$$
d\left(\left(a_{0}, b_{0}\right), \overline{\mathcal{C}}^{\mathbb{E}}\right) \leqslant \rho_{2}^{\mathbb{R}}\left(a_{0}, b_{0}\right) \text {, where } \rho_{2}^{\mathbb{R}}\left(a_{0}, b_{0}\right)=\min \left\{\left|s_{0}\right| / \mathcal{D}_{2}\left(a_{0}, b_{0}, s_{0}\right)=0 \text { and } s_{0} \in \mathbb{R}\right\} .
$$

Thus, the supremum of $\rho_{1}^{\mathbb{R}}(\boldsymbol{t}), \rho_{2}^{\mathbb{R}}(\mathfrak{a}, \boldsymbol{b})$ provides an upper bound of the Hausdorff distance; at least for those subsets of both curves where the considered minimums are well defined. Because of computational difficulties, in the examples below, instead of computing $\rho_{1}^{\mathbb{R}}(t), \rho_{2}^{\mathbb{R}}(a, b)$, we will minimize the module of all roots (not only real) of $\mathcal{D}_{1}\left(t_{0}, s\right)$ and $\mathcal{D}_{2}\left(a_{0}, b_{0}, s\right)$. That is, for $t_{0} \in \mathbb{R}$ and for $\left(a_{0}, b_{0}\right) \in \mathcal{C}^{\mathbb{P}}$ such that $\mathcal{D}_{1}\left(\mathfrak{t}_{0}, s\right)$ and $\mathcal{D}_{2}\left(a_{0}, b_{0}, s\right)$ are well defined, we will study

$$
\rho_{1}\left(t_{0}\right):=\min \left\{\left|s_{0}\right| / \mathcal{D}_{1}\left(t_{0}, s_{0}\right)=0\right\} \text { and } \rho_{2}\left(a_{0}, b_{0}\right):=\min \left\{\left|s_{0}\right| / \mathcal{D}_{2}\left(a_{0}, b_{0}, s_{0}\right)=0\right\},
$$

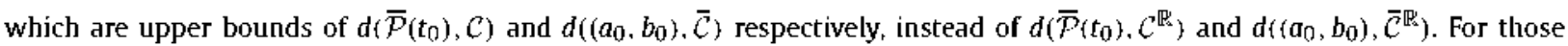
subsets of both curves, where the corresponding polynomials are well defined, we consider

$$
\Delta\left(\mathcal{C}^{\mathbb{E}}, \bar{C}^{\mathbb{E}}\right):=\max \left\{\sup _{P \equiv \mathcal{C} R}\{d(P, \bar{C})\}, \sup _{Q} \equiv \bar{C}^{\mathbb{R}}\{d(Q, \mathcal{C})\}\right\} .
$$

Note that this means that every real point on each of the curves is at distance, at most $\Delta\left(\mathcal{C}^{\mathbb{P}}, \bar{C}^{\mathbb{P}}\right)$, of a complex point on the other curve. Unfortunately, although it gives an idea of the closeness between of the curves, $\Delta\left(\mathcal{C}^{\mathbb{R}}, \overline{\mathcal{C}}^{\mathbb{E}}\right) \leqslant H\left(\mathcal{C}^{\mathbb{R}}, \overline{\mathcal{C}}^{\mathbb{E}}\right)$. Nevertheless, in the examples below we will look for empirical evidences indicating that the computed bound of $\Delta\left(\mathcal{C}^{\mathbb{R}}, \overline{\mathcal{C}}^{\mathbb{R}}\right.$.) also bounds $H\left(\mathcal{C}^{\mathbb{P}}, \overline{\mathcal{C}}^{\mathbb{R}}\right)$; for that we test empirically that, in our computations, $\rho_{1}\left(t_{0}\right)=\rho_{1}^{\mathbb{P}}\left(t_{0}\right), \rho_{2}\left(a_{0}, b_{0}\right)=\rho_{2}^{\mathbb{P}_{2}}\left(a_{0}, b_{0}\right)$.

In order to bound $\Delta\left(c^{\mathbb{R}}, \bar{c}^{\mathbb{R}}\right)$, we first prove the following lemma.

Lemma 6.2. Let $p(z)=a_{n} z^{n}+\cdots+a_{0} \in[z]$, with $a_{n} \neq 0$, and let $z_{1}, \ldots, z_{n}$ be its roots.

$$
\min _{1 \leqslant n}\left\{\left|z_{i}\right|\right\} \leqslant \min \left\{\left(\begin{array}{l}
n \\
j
\end{array}\right)\left|\frac{a_{0}}{a_{i}}\right|^{\frac{1}{t}} \text { where } a_{i} \neq 0 \text { and } 1 \leqslant i \leqslant n\right\} \text {. }
$$

Proof. The result is obvious if $a_{0}=0$. So, let $a_{0} \neq 0$, let $m=\min _{1}$ isn $\left\{\left|z_{i}\right|\right\}$, and let $a_{i} \neq 0$ with $1 \leqslant i \leqslant n$. We prove that $m \leqslant\left(\begin{array}{l}n \\ j\end{array}\right) \mid \frac{a_{0}}{a_{j}} \frac{1}{j}$. We express $p(z)$ as $p(z)=a_{n}\left(z^{n}-\sigma_{1} z^{n-1}+\sigma_{2} z^{n-2}+\cdots+(-1)^{n} \sigma_{n}\right)$, where $\sigma_{1}, \ldots, \sigma_{n}$ are the elementary symmetric functions of $z_{1}, \ldots, z_{n}$. If $i<n$ then

$$
\begin{aligned}
\left|\frac{a_{0}}{a_{i}}\right|=\frac{\left|\sigma_{n}\right|}{\left|\sigma_{n-i}\right|} & =\frac{\left|z_{1} \cdots z_{n}\right|}{\left|z_{1} \cdots z_{n-i}+\cdots+z_{i+1} \cdots z_{n}\right|} \geqslant \frac{\left|z_{1} \cdots z_{n}\right|}{\left|z_{1} \cdots z_{n-i}\right|+\cdots+\left|z_{i+1} \cdots z_{n}\right|} \\
& =\frac{1}{\frac{1}{\left|z_{n-i+1} \cdots z_{n}\right|}+\cdots+\frac{1}{\left|z_{1} \cdots z_{j}\right|}} \geqslant \frac{1}{\left(\begin{array}{c}
n \\
i
\end{array}\right) \frac{1}{m}} .
\end{aligned}
$$

If $i=n$ then $\left|a_{0} / a_{n}\right|=\left|z_{1} \cdots z_{n}\right| \geqslant m^{n}$.

In the sequel, we denote the coefficients of $\mathcal{D}_{1}$ and $\mathcal{D}_{2}$ as (note that $\operatorname{deg}_{s}\left(\mathcal{D}_{1}\right)=\operatorname{deg}_{s}\left(\mathcal{D}_{2}\right)=\operatorname{deg}(\mathcal{C})=\operatorname{deg}(\overline{\mathcal{C}})$ )

$$
\mathcal{D}_{1}(t, s)=A_{n}(t) s^{n}+\cdots+A_{0}(t), \quad \mathcal{D}_{2}(a, b, s)=B_{n}(a, b) s^{n}+\cdots+B_{0}(a, b) .
$$

Then, lemma 6.2 implies the following corollaries: 
Corollary 6.3. Let to $\in \mathbb{R}$, and $(a, b) \in \mathcal{C}^{\mathbb{R}}$ be such that $\mathcal{D}_{1}\left(t_{0}, s\right)$ and $\mathcal{D}_{2}\left(a_{0}, b_{0}, s\right)$ are well defined. Then,

1. $d\left(\overline{\mathcal{P}}\left(t_{0}\right), \mathcal{C}\right) \leqslant \min \left\{\left(_{j}^{n}\right)\left|\frac{A_{0}\left(t_{0}\right)}{A_{j}\left(t_{0}\right)}\right|^{\frac{1}{1}}\right.$ where $A_{i}\left(t_{0}\right) \neq 0$ and $\left.1 \leqslant i \leqslant n\right\}$.

2. $d\left(\left\{a_{0}, b_{0}\right), \bar{C}\right) \leqslant \min \left\{\left(q_{i}^{n}\right)\left|\frac{B_{0}\left(a_{0}, b_{0}\right)}{B_{1}\left(a_{0}, b_{0}\right)}\right|^{\frac{1}{t}}\right.$ where $B_{i}\left(a_{0}, b_{0}\right) \neq 0$ and $\left.1 \leqslant i \leqslant n\right\}$.

In addition, using the expression of the coefficients given by the Taylor expansion, the next corollary also holds.

Corollary 6.4. Let $t_{0} \in \mathbb{R}$, and $\left(a_{0}, b_{0}\right) \in \mathcal{C}^{\mathbb{R}}$ such that $\mathcal{D}_{1}\left(t_{0}, s\right)$ and $\mathcal{D}_{2}\left(a_{0}, b_{0}, s\right)$ are well defined. Then,

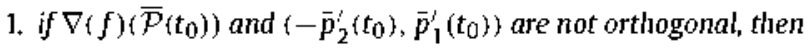

$$
d\left(\overline{\mathcal{P}}\left(t_{0}\right), \mathcal{c}\right) \leqslant n\left|\frac{f\left(\overline{\mathcal{P}}\left(t_{0}\right)\right)}{\nabla(f)\left(\overline{\mathcal{P}}\left(t_{0}\right)\right) \cdot\left(-\bar{p}_{2}^{\prime}\left(t_{0}\right), \bar{p}_{1}^{\prime}\left(t_{0}\right)\right)}\right|\left\|\left(-\bar{p}_{2}^{\prime}\left(t_{0}\right), \bar{p}_{1}^{\prime}\left(t_{0}\right)\right)\right\|_{2},
$$

2. if $\nabla(\bar{f})\left(a_{0}, b_{0}\right)$ and $\nabla(f)\left(a_{0}, b_{0}\right)$ are not orthogonal, then

$$
d\left(\left(a_{0}, b_{0}\right), \overline{\mathcal{C}}\right) \leqslant n\left|\frac{\bar{f}\left(a_{0}, b_{0}\right)}{\nabla(\bar{f})\left(a_{0}, b_{0}\right) \cdot \nabla(f)\left(a_{0}, b_{0}\right)}\right|\left\|\nabla(f)\left(a_{0}, b_{0}\right)\right\|_{2} .
$$

In the next examples we apply these results.

Example 6.5 (A compact curve example). We consider $\epsilon=\frac{1}{100}$ and the compact real curve $\mathcal{C}$

$$
\begin{aligned}
f(x, y)= & \frac{27399}{27397} y^{2}+\frac{20000001}{20000000} y^{3}+\frac{21}{100} y^{4}+\frac{27399}{27397} x y-\frac{3335608}{399953} x y^{2}+x y^{3}+\frac{27399}{27397} x^{2}+\frac{935993}{399997} x^{2} y \\
& +\frac{74}{25} x^{2} y^{2}-\frac{19999999}{20000000} x^{3}-\frac{71}{50} x^{3} y+\frac{1}{4} x^{4}+\frac{1}{125} x+\frac{1}{125} y .
\end{aligned}
$$

The output curve $\overline{\mathcal{C}}$ and its parametrization are

$$
\begin{aligned}
& \bar{f}(x, y)= x y^{3}+0.01055547819 x+0.005727140309 y+1.001749792 y^{2}+0.9870028562 y^{3}+0.21 y^{4} \\
&+0.9912384503 x^{2}-0.9982833629 x^{3}+0.25 x^{4}+1.053103489 x y-8.391588270 x y^{2} \\
&+2.341024446 x^{2} y+2.96 x^{2} y^{2}-1.42 x^{3} y+0.00002796854235 \\
& \overline{\mathcal{P}}(t)=\left(\frac{\bar{p}_{1}(t)}{\bar{q}(t)}, \frac{\bar{p}_{2}(t)}{\bar{q}(t)}\right)
\end{aligned}
$$

where

$$
\begin{aligned}
\bar{p}_{1}(t)= & 1.730167858 \cdot 10^{293} t+4.334613757 \cdot 10^{292} t^{3}+5.424023201 \cdot 10^{291} t^{4} \\
& +8.641660646 \cdot 10^{292}+1.299004236 \cdot 10^{293} t^{2} . \\
\bar{p}_{2}(t)= & -1.039366064 \cdot 10^{292} t^{2}-4.319011339 \cdot 10^{290} t^{4}-6.947686841 \cdot 10^{291} \\
& -3.459888148 \cdot 10^{291} t^{3}-1.387682118 \cdot 10^{292} t, \\
\bar{q}(t)= & 1.125103592 \cdot 10^{299} t^{2}+3.749748302 \cdot 10^{292} t^{3}+7.503078839 \cdot 10^{292} \\
& +4.686439171 \cdot 10^{291} t^{4}+1.500376938 \cdot 10^{293} t .
\end{aligned}
$$

We first observe that the polynomial $\mathcal{D}_{1}\left(t_{0}, s\right)$ is well defined for every $t_{0} \in \mathbb{R}$. In this situation, in order to bound $\rho_{1}(t)$ we maximize the function $R_{1}(t)=4\left|\frac{A_{0}(t)}{A_{1}(t)}\right|$ (see Corollary 6.3 ). $R_{1}(t)$ is continuous in $\mathbb{R}\left\{\left\{\alpha_{1}, \alpha_{2}\right\}\right.$, where $\alpha_{j}$ are real zeros of the denominator that are isolated in the intervals $I_{1}=(-2.020041475,-2.014041475), I_{2}=(-1.998980609,-1.998920609)$. Then,

$$
\max \left\{R_{1}(\mathrm{f}) \mid t \in \mathbb{R} \backslash\left(I_{1} \cup I_{2}\right)\right\}=0.2511290220 .
$$

In order to bound $d\left(\mathcal{P}\left(t_{0}\right), \overline{\mathcal{C}}\right.$ ), when $t_{0} \in I_{1} \cup I_{2}$, we consider $R_{2}(t)=\left(\begin{array}{l}4 \\ 2\end{array}\right) \sqrt{\mid \frac{A_{0}(t)}{A_{2}(t)}}$ (see Corollary 6.3 ). $R_{2}(t)$ is continuous in the adherence of $I_{1} \cup I_{2}$, and its maximum is 1.843001438 . Therefore, we conclude that

$$
\rho_{1}(t) \leqslant 1.843001438 \text {. }
$$


Now, we perform some empirical tests to show evidences that $\rho_{1}(t)=\rho_{1}^{\mathbb{R}}(t)$. First, let $D_{1}(s)=\lim _{t \mapsto \pm \infty} \mathcal{D}_{1}(t, s)$. Then,

$$
\min \left\{\left|s_{0}\right| / D_{1}\left(s_{0}\right)=0 \text { and } s_{0} \in \mathbb{R}\right\}=\min \left\{\left|s_{0}\right| / D_{1}\left(s_{0}\right)=0\right\}=0.004018853976 .
$$

Since the roots of a polynomial depend continuously on its coefficients, for every $\delta>0$ there exists $K>0$ such that for all $\left|t_{0}\right|>K$ there is a root $s_{0}$ of $\mathcal{D}_{1}\left(t_{0}, s\right)$ with $\left\|0.004018853976-s_{0}\right\|_{2}<\delta$. It might happen that these roots are all complex. However, in our example, we see that

$$
0.004018853976 \leqslant \rho_{1}\left((-10)^{i}\right)=\rho_{1}^{\mathbb{R}}\left((-10)^{i}\right) \leqslant 0.004023539023
$$

for $i=1, \ldots, 20$; reaching the lower bound from $i=12$. Next, for each real pole of $R_{1}(t)$ as well as for each real critical value of $R_{1}(t)$, we consider a sequence of isolating intervals $\mathrm{Ji}$ of length $1 / 10^{i+5}$, we take the middle point $t_{i}$, and we analyze $\rho_{1}\left(t_{i}\right), \rho_{1}^{\mathbb{R}}\left(t_{i}\right)$. For each of the two poles we get

$$
\rho_{1}\left(t_{i}\right)=\rho_{1}^{\mathbb{F}_{R}}\left(\mathbf{t}_{i}\right) \leqslant 0.07620545140
$$

for $i=1, \ldots, 20$; reaching the equality from $i=11$. For each of the eight critical values we get

$$
\rho_{1}\left(t_{i}\right)=\rho_{1}^{\mathbb{R}}\left(\mathfrak{t}_{i}\right) \leqslant 0.05039734676
$$

for $i=1, \ldots, 20$; reaching the equality from $i=11$.

$\mathcal{D}_{2}(a, b, s)$ is well defined for all $(a, b) \in \mathcal{C}^{\mathbb{R}}$. In order to bound $\rho_{2}(a, b)$ one may maximize the function $4\left|\frac{B_{0}(a, b)}{B_{1}(a, b)}\right|$ (see Corollary 6.3) under the constrain $f(a, b)=0$, for instance using Lagrange multipliers. Here, we simply show evidences for $\rho_{2}(a, b)$ being small and for $\rho_{2}(a, b)=\rho_{2}^{\mathbb{R}}(\boldsymbol{a}, b)$. First we observe that $\mathcal{C}^{\mathbb{P}} \subset[-9 / 512,8041 / 1024] \times$ $[-20057 / 1024,2117 / 1024]$. Now, for $i=2, \ldots, 100$ we consider the partition $\Delta_{i}:=\left\{a_{i, j}:=\alpha_{1}+\frac{j}{j}\left(\alpha_{2}-\alpha_{1}\right\}_{0<j<i}\right.$ of the open interval $\left(\alpha_{1}, \alpha_{2}\right)=\{-9 / 512,8041 / 1024)$. Next, for each $i$ we compute the set $s_{i}$ of intersections of $\mathcal{C}^{\mathbb{R}}$ with the line $x=a_{i, j}$. We obtain $m_{i}^{\mathbb{P}}:=\max \left\{\rho_{2}^{\mathbb{P}}(a, b) \mid\{a, b) \in \Omega_{i}\right\}$ and $m_{j}:=\max \left\{\rho_{2}(a, b) \mid(a, b) \in \Omega_{i}\right\}$, and we check whether $m_{i}^{\mathbb{R}}=m_{i}$. Finally, we compute $m:=\max \left\{m_{i} \mid i=2, \ldots, 100\right\}$. We get that $m_{i}^{\mathbb{R}}=m_{i}$ for all $i$ with the exception of $i=63$ and $i=88$ where $m_{63}^{\mathbb{R}}=0.06012962586<0.06012962587=m_{63}, m_{88}^{\mathbb{R}}=0.04097517998>0.04097517997=m_{88}$. Moreover, $m=0.06109662080$.

As a conclusion, in this example, the computed bound for the distance between the curves is 1.843001438 .

Example 6.6 ( $A$ non-compact curve example). We consider $\epsilon=\frac{1}{100}$ and the non-compact real curve $\mathcal{C}$

$$
\begin{aligned}
f(x, y)= & \frac{30771}{30769} y^{2}+\frac{3571429}{3571428} y^{3}+y^{4}+\frac{30771}{30769} x y-\frac{20535699}{1785713} x y^{2}+x y^{3}+\frac{22919}{30154} x^{2}+\frac{409991}{71427} x^{2} y \\
& +\frac{369}{100} x^{2} y^{2}-\frac{108569}{142854} x^{3}-\frac{78}{25} x^{3} y+\frac{19}{100} x^{4}+\frac{1}{100} x+\frac{1}{100} y .
\end{aligned}
$$

The output curve $\bar{C}$ and its parametrization are

$$
\begin{aligned}
& \bar{f}(x, y)= 1.037665836 y^{2}+0.9541760347 y^{3}+y^{4}+0.7557889914 x^{2}-0.7606067226 x^{3}+0.19 x^{4}+x y^{3} \\
&+0.01080256798 x+0.009723389264 y+1.033610692 x y-11.58684298 x y^{2}+5.792835786 x^{2} y \\
&+3.69 x^{2} y^{2}-3.120000001 x^{3} y+0.00004058975565 \\
& \overline{\mathcal{P}}(t)=\left(\frac{\bar{p}_{1}(t)}{\bar{q}(t)} \cdot \frac{\bar{p}_{2}(t)}{\bar{q}(t)}\right)
\end{aligned}
$$

where

$$
\begin{aligned}
\bar{p}_{1}(t)= & 5.634074231 \cdot 10^{345}+8.492460682 \cdot 10^{345} t^{2}+3.555839494 \cdot 10^{344} t^{4} \\
& +2.837737358 \cdot 10^{345} t^{3}+1.129568064 \cdot 10^{346} t \\
\bar{p}_{2}(t)= & -6.907472833 \cdot 10^{339} t^{4}-1.112114026 \cdot 10^{341}-5.534739853 \cdot 10^{340} t^{3} \\
& -2.220814182 \cdot 10^{341} t-1.663027642 \cdot 10^{341} t^{2} \\
\bar{q}(t)= & 1.423745556 \cdot 10^{341} t^{4}+1.138797024 \cdot 10^{342} t^{3}+4.553560207 \cdot 10^{342} t \\
& +3.415784668 \cdot 10^{342} t^{2}+2.276365040 \cdot 10^{342}
\end{aligned}
$$

We first observe that the polynomial $\mathcal{D}_{1}\left(t_{0}, s\right)$ is well defined for every $t_{0} \in \mathbb{R}_{k}$ with the exception of the two real poles $\beta_{1}, \beta_{2}$ of $\overline{\mathcal{P}}(t)$, that are isolated in the intervals $(-1.997680664,-1.997619629),(-2.008911133,-2.008850098)$. In this situation, in order to bound $\rho_{1}(t)$ when $t \in \mathbb{R} \backslash\left\{\beta_{1}, \beta_{2}\right\}$, we maximize the function $R_{1}(t)=4\left|\frac{A_{0}(t)}{A_{1}(t)}\right|$ (see Corol- 
lary 6.3). $R_{1}(t)$ is continuous in $\mathbb{R}\left\{\left\{\alpha_{1}, \alpha_{2}\right\}\right.$, where $\alpha_{i}$ are real zeros of the denominator that are isolated in the intervals $I_{1}=(-1.996163599,-1.996123599), I_{2}=(-2.034359611,-2.020359611)$. Then,

$$
\max \left\{R_{1}(\mathrm{t}) \mid t \in \mathbb{R} \backslash\left(I_{1} \cup I_{2}\right)\right\}=0.301275147 .
$$

In order to bound $d\left(\mathcal{P}_{\left(t_{0}\right)}, \overline{\mathcal{C}}\right.$ ), when $t_{0} \in I_{1} \cup I_{2}$, we consider $R_{2}(t)=\left({ }_{2}^{4}\right) \sqrt{\left|\frac{A_{0}(t)}{A_{2}(t)}\right|}$ (see Corollary 6.3 ). $R_{2}(t)$ is continuous in the adherence of $I_{1} \cup I_{2}$, and its maximum is 1.987657564 . Therefore, we conclude that

$$
\forall t \in \mathbb{R} \backslash\left\{\alpha_{1}, \alpha_{2}\right\}, \quad \rho_{1}(t) \leqslant 1.987657564 .
$$

Now, we perform some empirical tests to show evidences that $\rho_{1}(t)=\rho_{1}^{\text {Fit }}(t)$. First we analyze the behavior through the real asymptotes. The real asymptotes of $\mathcal{C}$ and $\bar{C}$ are, respectively:

$$
\begin{array}{ll}
\mathcal{L}_{1}:=x-15.11127611 y-2.469501937, & \mathcal{L}_{2}:=x-1.659899985 y+0.6395629633, \\
\overline{\mathcal{L}}_{1}:=x-15.11127611 y-2.483911806, & \overline{\mathcal{L}}_{2}:=x-1.659899985 y+0.6343579098
\end{array}
$$

Moreover,

$$
H\left(\mathcal{L}_{1}, \overline{\mathcal{L}}_{1}\right)=0.0009515027113, \quad H\left(\mathcal{L}_{2}, \overline{\mathcal{L}}_{2}\right)=0.002685992105
$$

Next, for each of the two real poles $\beta_{1}, \beta_{2}$ of $\overline{\mathcal{P}}(t)$ we consider a sequence of isolating intervals $J_{i}\left(\beta_{j}\right)$ of length $1 / 10^{5 i}$, we take the middle point $t_{i, \beta_{j}}$, and we analyze $\rho_{1}\left(t_{i, \beta_{j}}\right), \rho_{1}^{\mathbb{R}}\left(t_{i, \beta_{j}}\right)$. We get, for $i=1, \ldots, 40$

$$
\begin{aligned}
& \rho_{1}\left(t_{i, \beta_{1}}\right)=\rho_{1}^{\mathbb{R}}\left(t_{i, \beta_{1}}\right)=0.000951502783>0.0009515027113=H\left(\mathcal{L}_{1}, \overline{\mathcal{L}}_{1}\right), \\
& \rho_{1}\left(t_{i, \beta_{2}}\right)=\rho_{1}^{\mathbb{R}}\left(t_{i, \beta_{2}}\right)=0.00268599101<0.002685992105=H\left(\mathcal{L}_{2}, \overline{\mathcal{L}}_{2}\right) .
\end{aligned}
$$

Now, let $D_{1}(s)=\lim _{t \mapsto \pm \infty} \mathcal{D}_{1}(t, s)$. Then,

$$
\min \left\{\left|s_{0}\right| / D_{1}\left(s_{0}\right)=0 \text { and } s_{0} \in \mathbb{R}\right\}=\min \left\{\left|s_{0}\right| / D_{1}\left(s_{0}\right)=0\right\}=0.001918863706 .
$$

Furthermore, in our example, we see that

$$
0.001918863706 \leqslant \rho_{1}\left((-10)^{i}\right)=\rho_{1}^{\mathbb{R}}\left((-10)^{i}\right) \leqslant 0.001922644324
$$

for $i=1, \ldots, 20$; reaching the lower bound from $i=8$. Next, for each real pole of $R_{1}(t)$ as well as for each real critical value of $R_{1}(t)$, we consider a sequence of isolating intervals $J_{i}$ of length $1 / 10^{i+5}$, we take the middle point $t_{i}$, and we analyze $\rho_{1}\left(t_{i}\right), \rho_{1}^{\mathbb{F}}\left(t_{i}\right)$. For each of the two poles we get

$$
\rho_{1}\left(t_{i}\right)=\rho_{1}^{\mathbb{F}}\left(\mathbf{t}_{i}\right) \leqslant 0.05760637790
$$

for $i=1, \ldots, 20$; reaching the equality from $i=11$. For each of the eight critical values we get

$$
\rho_{1}\left(t_{i}\right)=\rho_{1}^{\mathbb{F}}\left(\mathbf{t}_{\mathfrak{f}}\right) \leqslant 0.07103885930
$$

for $i=1, \ldots, 20$; reaching the equality from $i=17$.

$\mathcal{D}_{2}(a, b, s)$ is well defined for all $(a, b) \in \mathcal{C}^{\mathbb{P}}$. In order to bound $\rho_{2}(a, b)$ one may maximize the function $4\left|\frac{g_{0}(a, b)}{B_{1}(a, b)}\right|$ (see Corollary 6.3) under the constrain $f(a, b)=0$, for instance using lagrange multipliers. Here, we simply show evidences for $\rho_{2}(a, b)$ being small and for $\rho_{2}(a, b)=\rho_{2}^{\mathbb{F}}(a, b)$. For each natural number $i$ we consider the two intersection points of $\mathcal{C}^{\mathbb{R}}$ and $x=i$, say $\left(i, b_{1}\right),\left(i, b_{2}\right)$. For each of these points we compute the corresponding $\rho_{2}^{\mathbb{F}}\left(i, b_{j}\right)$. We repeat this process till $\left|\rho_{2}^{\mathbb{P}}\left(i, b_{1}\right)-0.000951502783\right|<10^{-6},\left|\rho_{2}^{\mathbb{R}}\left(i, b_{2}\right)-0.00268599101\right|<10^{-6}$ (see above for the meaning of 0.000951502783 and 0.00268599101 ). We perform this experiment also for $x=-i$, for $y=i$, and for $y=-i$. The result is $[-3430,3431]$ for the $x$ and $[-2067,2068]$ for $y$. In this situation, we empirically consider that out of the compact $B=[-3430,3431] \times[-2067,2068]$, the curves behave as the asymptotes, and we analyze the distance in $B$. For this purpose, as in Example 6.5, for $i=7000, \ldots, 7010$ we consider the partition $\Delta i:=\left\{a_{i, j}:=\alpha_{1}+\frac{j}{j}\left(\alpha_{2}-\alpha_{1}\right)\right\}_{0<j<i}$ of the open interval $\left(\alpha_{1}, \alpha_{2}\right)=(-3430,3431)$. Next, for each $i$ we compute the set $\Omega_{i}$ of intersections of $\mathcal{C}^{\mathbb{R}}$ with the line $x=a_{i, j}$. We obtain $m_{i}^{\mathbb{R E}}:=\max \left\{\rho_{2}^{\mathbb{R}}(a, b) \mid(a, b) \in \Omega_{i}\right\}$ and $m_{i}:=\max \left\{\rho_{2}(a, b) \mid(a, b) \in \Omega_{i}\right\}$, and we check whether $m_{j}^{\mathbb{R}}=m_{i}$. Finally, we compute $m:=\max \left\{m_{i} \mid i=7000, \ldots, 7010\right\}$. We get that $m_{i}^{\mathbb{R}}=m_{i}$ and $m=0.03416457806$.

As a conclusion, in this example, the computed bound for the distance between the curves is 1.987657564 .

As a final summary, let us mention that, in both examples, $\epsilon=0.01$ and the computed bounds for the distance were 1.843001438 and 1.987657564 , respectively. These two final bounds were derived applying Corollary 6.3 , although all the other partial bounds empirically computed were much smaller. 


\section{References}

Alcázat, J.G., Sendra, J.R., 2005. Computation of the topology of real algebraic space curves, Journal of Symbolic Computation 39 (6), $719-744$.

Aliprantis, C.D., Border, $K_{+} C_{+}$2006. Infinite Dimensional Analysis, Springer-Verlag.

Bajaj. C., Royappa, A., 2000. Parameterization in finite precision. Algorithmica 27 (1), 100-114.

(oolidge. J.L., 1950. A Treatise on Algebraic Plane Curves. Dover, New York.

Corless, R.M., Gianni, P.M. Trager, B.M., Watt, S.M., 1995. The singular value decomposition for polynomial systems. In: Proc. ISSAC 1905. ACM Press. pp. $195-207$.

Corless, R.M., Giesbrecht, M.W., Kotsireas, IS., Watt, S.M., 2000. Numerical implicitization of parametric hypersurfaces with linear algebra. In: Proc. Artificial Intelligence with Symbolic Computation. AISC, 2000. In: LNAI, vol, 1930. Sptinger-Verlag, pp. 174-183.

Corless, R.M., Ciesbrecht, M.W., Kotsireas, I.S., van Hoeij. M., Watt, S.M., 2001. Towards factoring bivariate approximate polynomials. In: Mourrain, Bernard (Ed.). Proc. ISSAC 2001, London, Canada. ACM Press, New York, NY, USA, PP. 85-92.

Dokken, T., 2001. Approximate implicitization. In: Lyche. Tom, Schumakes, Larry L. (Eds.), Mathematical Methods in CAGD. Oslo, 2000. Vanderbilt University Press.

Emiris, I,Z., Galligo, A., Lombardi, H., 1997. Certified approximate univariate GCDs. Journal of Pure and Applied Algebra 117-118, 229-251.

Fatouki, R.T., Rajan, V.T, 1988. On the numerical condition of algebraic curves and surfaces. 1. Implicit equations. Computer Aided Geometric Design 5, 215-252.

Gahleitner. J., Jïttler, B., Schicho. J., 2002. Approximate parameterization of planar cubic curve segments. In: Proc. Fifth International Conference on Curves and Surfaces. Saint-Malo, 2002. Nashboro Press, Nashville, TN, PP. 1-13.

Calligo. A., Rupprech, D., 2002. Irreducible decomposition of curves, Journal of Symbolic Computation 33, 661-677.

Hartmanin, E., 2000. Numerical parameterization of curves and surfaces. Computer Aided Geometry Design 17, $251-266$.

Kaltofen, E., May, J.P., Yang, Z, Zhi, L., 2008. Approximate factorization of multivariate polynomials using singular value decomposition. Journal of Symbolic Computation 43 (5), 359-376.

Mĭuk, $M_{*}$ Sendta, J.R., Winkler, $F_{*}$ 1997. On the complexity of parametrizing curves, Beiltrage zur Algebra und Geometrie 37 (2), $309-328$.

Pan, V.Y., 1996. Numerical computation of a polynomial GCD and extensions. Tech. report, No. 2069, Sophia-Antipolis, France.

Pan, V.Y., 2001. Univariate polynomials: Nearly optimal algorithms for factorization and rootfinding. In: Mourrain, Bernard (Ed.), ISSAC 2001, London, Canada. ACM Press, New York, NY, USA, pp. 253-267.

Pérez-Diaz, S., Sendra, J., Sendra, J.R, 2004, Parametrization of approximate algebraic curves by lines. Theoretical Computer Science 315 (2-3), 627-650.

Pérez-Díaz, S, Sendra, J, Sendta, J.R, 2005. Parametrization of approximate algebraic surfaces by lines. Computer Aided Geometries Design 22 (2). 147-181.

Pérez-Díaz, S., Sendra, J., Sendra, J.R, 2006. Distance bounds of $\epsilon$-points on hypersutfaces. Theoretical Computer Science $359,344-368$.

Pérez-Díaz, S., Rueda, S.L., Sendra. J., Sendra, J.R., 2009. Approximate parametrization of plane algebraic curves by linear systems of curves. arXiv: $0001.0320 \mathrm{v} 1$.

San Segundo, F., Sendra, J.R., 2005. Degree formulae for off5et curves. Journal of Pure and Applied Algebra 195, 301-335.

Sasaki, T., 2001. Approximate multivariate polynomial factorization based on zero-sum relations. In: Mourrain, Bernard (Ed.), Proc. ISSAC 2001, London. Canada, ACM Ptess, New York, NY, USA, pp. 284-291.

Sasaki, T., Terui, A., 2002. A formula for separating small roots of a polynomial. ACM, SIGSAM Bulletin 36 (3), $19-23$.

Sendra, J,R, 2002. Normal parametrizations of algebraic plane curves. Journal of Symbolic Computation 33, $863-885$.

Sendra, J.R., Winkler, J.R., Pérez-Diaz, S., 2007. Rational Algebraic Curves: A Computer Algebra Approach. Algorithms and Computation in Mathematics. vol. 22. Springer-Verlag, Heidelberg.

Winkler, F., 1996. Polynomial Algorithms in Computer Algebra. Springer-Verlag. Wien, New York 